\title{
ON CONFORMALLY INVARIANT SUBSETS OF THE PLANAR BROWNIAN CURVE
}

\author{
Vincent BEFFARA \\ Département de mathématiques, Université Paris-Sud, 91405 Orsay cedex, France
}

Received 8 June 2001, accepted 14 January 2003

ABSTRACT. - We define and study a family of generalized non-intersection exponents for planar Brownian motion: For each $A \subset \mathbb{C}$ we define an exponent $\xi(A)$ describing the decay of a non-intersection probability. Each of these exponents corresponds to a geometrically defined subset of the Brownian curve with dimension $2-\xi(A)$. As a consequence of this and continuity of $\xi(A)$ as a function of $A$, we prove the almost sure existence of pivoting points on a planar Brownian path.

(c) 2003 Éditions scientifiques et médicales Elsevier SAS

RÉSUMÉ. - Nous définissons et étudions une famille d'exposants de non-intersection généralisés pour le mouvement brownien plan : pour tout $A \subset \mathbb{C}$ nous définissons un exposant $\xi(A)$ décrivant la décroissance d'une probabilité de non-intersection. À chacun de ces exposants correspond une partie de la courbe brownienne définie de manière géométrique et dont la dimension est $2-\xi(A)$. Une conséquence de cette relation et de la continuité de $\xi(A)$ comme fonction de $A$ est l'existence presque sûre de points pivots sur la trajectoire brownienne. () 2003 Éditions scientifiques et médicales Elsevier SAS

\section{Introduction}

Theoretical physicists have conjectured for more than twenty years that conformal invariance plays an important role in understanding the behaviour of critical twodimensional models of statistical physics. They justify by a mathematically non-rigorous argument involving renormalization, that in the scaling limit these models behave in a conformally invariant way; they have then been able to classify them via a real-valued parameter corresponding to the central charge of the associated Virasoro algebra, and to predict the exact value of critical exponents that describe the behaviour of these systems. Different models (for instance, self-avoiding walks and percolation) with the same central charge have the same exponents.

Recently, Schramm [20] introduced a family of new mathematical objects that give insight into these conjectures. These are random, set-valued, increasing processes 
$\left(K_{t}\right)_{t \geqslant 0}$ which he named Stochastic Loewner Evolution processes. For each positive number $\kappa$, there exists one such process of parameter $\kappa$, denoted $S L E_{\kappa}$. He proved that for various models, if they have a conformally invariant scaling limit, then this limit can be interpreted in terms of one of the $S L E_{\kappa}$ 's - the parameter $\kappa$ would then be related to the central charge of the model. One can then interpret the conjectures of the theoretical physicists in terms of properties of SLE.

In particular, Lawler, Schramm and Werner $[14,15]$ showed that for one specific value of the parameter $\kappa$ (namely $\kappa=6$ ) which conjecturally corresponded to the scaling limit of percolation cluster interfaces, the $S L E_{6}$ has the remarkable restriction property which relates its critical exponents to the so-called intersection exponents of planar Brownian motions. This led [14-17] to the derivation of the exact value of the intersection exponents between planar Brownian paths. Furthermore, it was later shown [24] that, in fact, the outer boundary of a planar Brownian curve has exactly the same law as that of an $S L E_{6}$. In other words, the geometry of critical two-dimensional percolation clusters in their scaling limit should be exactly that of a planar Brownian outer frontier.

In a very recent paper, Smirnov [21] showed that critical site percolation in the triangular lattice is conformally invariant in the scaling limit, so that the geometry of critical two-dimensional percolation cluster boundaries, in their scaling limit, is identical to that of a planar Brownian outer frontier.

Before all these recent developments, geometric properties of planar Brownian paths had already been the subject of numerous studies (see, e.g., [19] for references). In particular, the Hausdorff dimension of various geometrically defined subsets of the planar Brownian curve had been determined. For instance, Evans [5] showed that the Hausdorff dimension of the set of two-sided cone points of angle $\theta$ (i.e., points $B_{t}$ such that both $B_{[0, t]}$ and $B_{[t, 1]}$ are contained in the same cone of angle $\theta$ with endpoint at $B_{t}$ ) is $2-2 \pi / \theta$. In a series of papers (see, e.g., the review in [11]), Lawler proved that the dimension of various important subsets of the planar Brownian curve can be related to Brownian intersection exponents. In particular [9], he showed that the dimension of the set $C$ of cut points (i.e., points $B_{t}$ such that $B_{[0,1]} \backslash\left\{B_{t}\right\}$ is not connected) is $2-\xi$ where $\xi$ is the Brownian intersection exponent defined by

$$
p_{R}=P\left(B_{\left[0, T_{R}^{1}\right]}^{1} \cap B_{\left[0, T_{R}^{2}\right]}^{2}=\emptyset\right)=R^{-\xi+o(1)}
$$

(for independent Brownian paths $B^{1}$ and $B^{2}$ starting respectively from 1 and $-1, T_{R}^{1}$ and $T_{R}^{2}$ standing for their respective hitting times of the circle $\mathcal{C}(0, R)$ ).

In order to derive such results, and in particular the more difficult lower bound $d \geqslant$ $2-\xi$, the strategy is first to refine the estimate (1) to $p_{R} \asymp R^{-\xi}$ (we shall use this notation to denote the existence of two positive constants $c$ and $c^{\prime}$ such that $c R^{-\xi} \leqslant p_{R} \leqslant c^{\prime} R^{-\xi}$ ), then to derive second-moment estimates and finally to use these estimates to construct a random measure of finite $r$-energy supported on $C$, for all $r<2-\xi$. The determination of the values of the critical exponents via $S L E_{6}[14,15]$ then implies that the dimension of $C$ is 3/4. Similarly [8], the Hausdorff dimension of the outer frontier of a Brownian path can be interpreted in terms of another critical exponent, and the determination of this exponent using $S L E_{6}$ then implies (see [13] for a review) that this dimension is $4 / 3$ as conjectured by Mandelbrot. 
In the present paper we define and study a family of generalizations of the Brownian intersection exponent $\xi$ parameterized by subsets of the complex plane. For each $A \subset \mathbb{C}$, we define an exponent $\xi(A)$ as follows. Let $B^{1}$ and $B^{2}$ be two independent planar Brownian paths starting from uniformly distributed points on the unit circle. Then $\xi(A)$ is defined by

$$
p_{R}(A)=P\left(B_{\left[0, T_{R}^{1}\right]}^{1} \cap A B_{\left[0, T_{R}^{2}\right]}^{2}=\emptyset\right)=R^{-\xi(A)+o(1)}
$$

(with the notation $E_{1} E_{2}=\left\{x y: x \in E_{1}, \quad y \in E_{2}\right\}$ ). Note that the case $A=\{1\}$ corresponds to the usual intersection exponent. In Section 1 we first show that for a wide class of sets $A$

$$
p_{R}(A) \asymp R^{-\xi(A)} .
$$

In Section 2 we study regularity properties of the mapping $A \mapsto \xi(A)$. In particular we prove its uniform continuity (with respect to the Hausdorff metric) on certain families of sets. One important tool for this result is the fact that the constants implicit in (3) can in fact be taken uniform over these families of sets.

In Section 3 we associate to each set $A$ a geometrically defined subset $\mathcal{E}_{A}$ of the planar Brownian curve:

$$
\mathcal{E}_{A}=\left\{B_{t}: \exists \varepsilon>0,\left(B_{[t-\varepsilon, t]}-B_{t}\right) \cap A\left(B_{(t, t+\varepsilon]}-B_{t}\right)=\emptyset\right\} .
$$

Using the strong approximation and continuity of the mapping $A \mapsto \xi(A)$, we then show that the Hausdorff dimension of this subset of the planar Brownian curve is almost surely $2-\xi(A)$ when $\xi(A) \leqslant 2$ (and is 0 when $\xi(A)>2$ ). For example, when $A=\left\{\mathrm{e}^{\mathrm{i} \theta}, 0 \leqslant \theta \leqslant \alpha\right\}$ the corresponding subset $C_{\alpha}$ of the Brownian curve is the set of (local) pivoting points, i.e. points around which one half of the path can rotate by any angle smaller than $\alpha$ without intersecting the other half.

When $A \subset A^{\prime}$, then $\mathcal{E}_{A^{\prime}} \subset \mathcal{E}_{A}$. In particular, if $A$ contains 1 , then $\mathcal{E}_{A}$ is a subset of the set of (local) cut points and therefore the shape of the path in a neighbourhood of such a point is the same as that of the Brownian frontier in the neighbourhood of a cutpoint. This shows in particular that (at least some of) the exponents $\xi(A)$ also describe the Hausdorff dimension of sets of exceptional points of the scaling limit of critical percolation clusters.

In Section 4 we derive some bounds on the exponents $\xi(A)$ for small sets $A$, by a technique similar to that used by Werner [23] to estimate disconnection exponents. In particular, for small $\alpha$, we show that the exponent $\xi\left(C_{\alpha}\right)$ is strictly smaller than 2 , which implies the existence of pivoting points of any angle less than $\alpha_{0}>0$ on the planar Brownian curve. We then briefly present results of simulations which suggest that the maximal angle $\alpha_{0}$ is close to $3 \pi / 4$.

It is actually easy to define other "generalized" exponents in a similar fashion, by studying non-intersection properties between Brownian motions and some of their images under isometries and scalings, i.e. one can view $A$ as a subset of the linear group $\mathrm{GL}_{2}(\mathbb{R})$. One can also consider non-intersection properties between $B$ and its image $f(B)$ by a conformal map. For instance it is easy to see using the function $z \mapsto z^{2}$ that the exponent describing the non-intersection between $B$ and $-B$ is in fact twice the 
disconnection exponent. The methods of the present paper can then be adapted to such situations.

Similarly, one could extend the definitions to higher dimensions (the cases $d \geqslant 4$ can also be interesting if the set $A$ is sufficiently large), but conformal invariance then cannot be used, so that some of the tools in the present paper do not apply.

\section{Notations}

Throughout this paper, we will use the following notations for the asymptotic behaviour of positive functions (and sequences, with the same meaning):

- $f \sim g$ if $\lim _{t \rightarrow \infty}(f(t) / g(t))=1-$ and $f$ and $g$ are said to be equivalent;

- $f \approx g$ if $\log f \sim \log g$, i.e. if $\lim _{t \rightarrow \infty}(\log f(t)) /(\log g(t))=1-f$ and $g$ are then logarithmically equivalent;

- $f \asymp g$ if $f / g$ is bounded above and below, i.e. if there exist two positive finite constants $c$ and $C$ such that for all $t, c g(t) \leqslant f(t) \leqslant C g(t)$ - which we call strong approximation of $f$ by $g$.

\section{Generalized intersection exponents}

\subsection{Definition of the exponents}

PROPOSITION AND DEFINITION. - Let A be a non-empty subset of the complex plane and $B^{1}, B^{2}$ be two independent Brownian paths starting uniformly on the unit circle $\mathcal{C}(0,1)$; define the hitting time $T_{R}^{i}$ of $\mathcal{C}(0, R)$ by $B^{i}$ and let $\tau_{n}^{i}=T_{\exp (n)}^{i}$,

$$
\begin{aligned}
& E_{n}=E_{n}(A)=\left\{B_{\left[0, \tau_{n}^{1}\right]}^{1} \cap A B_{\left[0, \tau_{n}^{2}\right]}^{2}=\emptyset\right\}, \\
& q_{n}(A)=P\left(E_{n}\right) \text { and } p_{R}(A)=P\left(E_{\log R}\right) .
\end{aligned}
$$

Then, assuming the existence of positive constants $c$ and $C$ such that $p_{R}(A) \geqslant c R^{-C}$, there exists a real number $\xi(A)$ such that, when $R \rightarrow \infty$,

$$
p_{R}(A) \approx R^{-\xi(A)} .
$$

Proof. - This is a standard sub-multiplicativity argument. If $B$ is a Brownian path starting on $\mathcal{C}(0,1)$ with any law $\mu$, then the law of $B_{\tau_{1}(B)}$ on the circle $\mathcal{C}(0, e)$ has a density (relative to the Lebesgue measure) bounded and bounded away from zero by universal constants (i.e., independently of $\mu$ ). Combining this remark with the Markov property at the hitting times of the circle of radius $\mathrm{e}^{n}$ shows that:

$$
\forall m, n \geqslant 1 \quad q_{m+n} \leqslant c q_{n} q_{m-1} .
$$

Hence the family $\left(c q_{n-1}\right)$ is sub-multiplicative, and using Proposition 7 we have $q_{n} \approx$ $\mathrm{e}^{-\xi n}$, with $\xi \in(0, \infty)$, as well as a lower bound $q_{n} \geqslant c^{-2} \mathrm{e}^{-\xi(n+1)}$.

Remarks. - For some choices of $A$ there is an easy geometric interpretation of the event $E_{n}(A): \xi(\{1\})$ is the classical intersection exponent; if $A=(0, \infty)$, the $E_{n}(A)$ is the event that the paths stay in different wedges. 
If $A$ is such that no lower bound $p_{R}(A) \geqslant c R^{-C}$ holds, we let $\xi(A)=\infty$. However, in most of the results presented here, we will restrict ourselves to a class of sets $A$ for which it is easy to derive such lower bounds:

DEFINITION. - A non-empty subset A of the complex plane is said to be nice if it is contained in the intersection of an annulus $\{r<|z|<R\}($ with $0<r<R<\infty)$ with a wedge of angle strictly less than $2 \pi$ and vertex at 0 .

Indeed, let $A$ be such a set and let $\alpha<2 \pi$ be the angle of a wedge containing $A$ : $B^{1}$ and $A B^{2}$ will not intersect provided each path remains in a well-chosen wedge of angle $(2 \pi-\alpha) / 2$, and then it is standard to derive the following bound:

$$
p_{R}(A) \geqslant c R^{-4 \pi /(2 \pi-\alpha)} .
$$

The fact that $A$ be contained in an annulus will be needed in the following proof. The only usual case where this does not hold is when $A$ is a wedge itself; but in this case a direct study is possible, based on the derivation of cone exponents in [5] and the exact value of $\xi$ is then known (cf. next section for details).

We will often consider the case where $A$ is a subset of the unit circle. For such sets, $A$ is nice if and only if $\bar{A} \varsubsetneqq \partial \mathbb{U}$ (it is in fact easy to prove that for $A \subset \partial \mathbb{U}, \xi(A)=\infty$ if and only if $\bar{A}=\partial \mathbb{U}$ ).

\subsection{Strong approximation}

This whole subsection will be dedicated to the refinement of $p_{R} \approx R^{-\xi}$ into $p_{R} \asymp$ $R^{-\xi}$. This is not anecdotal, since this "strong" approximation will be needed on several occasions later.

THEOREM 1. - For every nice $A, p_{R}(A) \asymp R^{-\xi(A)}$, i.e. there exist positive constants $c(A)<C(A)$ such that

$$
c R^{-\xi(A)} \leqslant p_{R}(A) \leqslant C R^{-\xi(A)} .
$$

Moreover, the constants $c(A)$ and $C(A)$ can be taken uniformly on a collection $\mathcal{A}$ of subsets of the plane, provided the elements of $\mathcal{A}$ are contained in the same nice set.

Proof. - Note that since $A \in \mathcal{A}$ is nice, the exponents $\xi(A)$ exist and are uniformly bounded for $A \in \mathcal{A}$. The sub-additivity argument showed that $q_{n} \geqslant c \mathrm{e}^{-\xi(A) \cdot(n+1)}$, which implies readily the lower bound in the theorem. It is more difficult to derive the upper bound. By Proposition 7, it will be sufficient to find a finite constant $c_{-}(A)$ (that can be bounded uniformly for $A \in \mathcal{A}$ ) such that

$$
\forall n, n^{\prime} \quad q_{n+n^{\prime}} \geqslant c_{-} q_{n} q_{n^{\prime}} .
$$

In order to make the proof more readable, it is carried out here for a fixed $A$; however it is easy to see that, at each step, the constants can be taken uniformly for all $A$ contained in some fixed nice set $A_{0}$. Moreover, we shall first assume that $A_{0}$ is a subset of the unit circle: We briefly indicate at the end of the proof what are the few modifications needed to adapt it to the general case. 
The basic method is adapted from Lawler's proof for non-intersection exponents in [10], with some technical simplifications made possible using the absence of the $\lambda$ exponent. The main idea is to obtain a weak independence between the behaviour of the paths before and after they reach radius $\mathrm{e}^{n}$. The first step is an estimate concerning the probability that the paths are "well separated" when they reach radius $\mathrm{e}^{n}$ (more precisely, that they remain in two separated wedges between radius $\mathrm{e}^{n-1}$ and radius $\mathrm{e}^{n}$ ). Let $\mathcal{F}_{n}$ stands for the $\sigma$-field generated by both paths up to radius $\mathrm{e}^{n}$ (so that for instance $E_{n}$ is in $\mathcal{F}_{n}$ ).

LEMma (Technical). - Let $\eta>0$ and $\alpha<2 \pi-\eta$ such that $A$ is contained in a wedge of angle less than $\alpha$. Define

$$
W_{\alpha}=\left\{r \mathrm{e}^{\mathrm{i} \theta}: r>0,|\theta|<\frac{\alpha}{2}\right\},
$$

$\delta_{n}=\mathrm{e}^{-n}\left[\mathrm{~d}\left(B_{\tau_{n}^{1}}^{1}, A B_{\left[0, \tau_{n}^{2}\right]}^{2}\right) \wedge \mathrm{d}\left(A B_{\tau_{n}^{2}}^{2}, B_{\left[0, \tau_{n}^{1}\right]}^{1}\right)\right]$ and the following events:

$$
\begin{aligned}
& U_{n}^{1}=\left\{B_{\left[0, \tau_{n}^{1}\right]}^{1} \cap\left\{|z| \geqslant \mathrm{e}^{n-1}\right\} \subset-W_{2 \pi-\alpha-\eta}\right\}, \\
& U_{n}^{2}=\left\{A B_{\left[0, \tau_{n}^{2}\right]}^{2} \cap\left\{|z| \geqslant \mathrm{e}^{n-1}\right\} \subset W_{\alpha}\right\},
\end{aligned}
$$

and $U_{n}=U_{n}^{1} \cap U_{n}^{2}$. Then:

$$
\exists c, \beta>0 \forall \varepsilon>0 \forall r \in\left[\frac{3}{2}, 3\right] \quad P\left(E_{n+r}, U_{n+r} \mid \mathcal{F}_{n}, E_{n}, \delta_{n} \geqslant \varepsilon\right) \geqslant c \varepsilon^{\beta} .
$$

Proof. - The proof is easy and uses only simple estimates on Brownian Motion in a wedge, we omit the details.

We now prove that paths conditioned not to intersect up to radius $\mathrm{e}^{n+2}$ have a good chance to be well separated at this radius, uniformly with respect to their behaviour up to radius $\mathrm{e}^{n}$ :

LEMMA (End-separation). - There exists $c>0$ such that, for every $n>0$ :

$$
P\left(U_{n+2} \mid E_{n+2}, \mathcal{F}_{n}\right) \geqslant c
$$

(in other words, the essential lower bound of $P\left(U_{n+2} \mid E_{n+2}, \mathcal{F}_{n}\right)$, as an $\mathcal{F}_{n}$-measurable function, is not less than $c$ ).

Proof. - The technical lemma states that start-separation occurs if the starting points are sufficiently far from each other; more precisely, applying it for $r=2$, we obtain for all $\varepsilon>0$ :

$$
P\left(U_{n+2} \mid E_{n+2}, \mathcal{F}_{n}, \delta_{n} \geqslant \varepsilon\right) \geqslant c \varepsilon^{\beta} .
$$

Hence, what is to be proved is that two paths conditioned not to intersect have a positive probability to be far from each other after a relatively short time. To prove this fact, one has to use conditioning on the value of $\delta_{n}$. 
Fix $k>0$, and assume that $2^{-(k+1)} \leqslant \delta_{n}<2^{-k}$; let $\tau_{k}$ be the smallest $r$ such that one of the following happens: either $\delta_{n+r} \geqslant 2^{-k}$, or $E_{n+r}$ does not hold. It is easy to use scaling to prove that for some $\lambda>0$,

$$
P\left(\tau_{k} \geqslant 2^{-k}\right) \leqslant 2^{-\lambda},
$$

meaning that with positive probability (independent of $k$ and $n$ ) the paths separate or meet before reaching radius $\mathrm{e}^{n+2^{-k}}$. Hence, by the strong Markov property applied $k^{2}$ times, this leads to

$$
P\left(\tau_{k} \geqslant k^{2} 2^{-k} \mid 2^{-(k+1)} \leqslant \delta_{n}<2^{-k}\right) \leqslant 2^{-\lambda k^{2}} .
$$

The technical lemma states that $P\left(E_{n+2} \mid \delta_{n} \geqslant 2^{-(k+1)}\right) \geqslant c 2^{-\beta k}$ : combining both estimates then leads to

$$
P\left(\tau_{k} \geqslant k^{2} 2^{-k} \mid E_{n+2}, \delta_{n} \geqslant 2^{-(k+1)}\right) \leqslant c 2^{\beta k-\lambda k^{2}} .
$$

Consider now a generic starting configuration at radius $\mathrm{e}^{n}$, satisfying $E_{n}$ and hence $\delta_{n}>0$. Fix also $k_{0}>0$ and introduce the radii $\tau_{k}\left(\right.$ for $k_{0} \leqslant k<\infty$ ) defined by

$$
\tau_{k}=\operatorname{Inf}\left\{r: \delta_{n+r} \geqslant 2^{-k}\right\}
$$

(so that $\tau_{k}=0$ as long as $2^{-k} \leqslant \delta$ ). Eq. (8) can be rewritten (using the fact that the technical lemma is valid for all $r \geqslant 3 / 2$ ) as

$$
P\left(\tau_{k}-\tau_{k+1} \geqslant k^{2} 2^{-k} \mid E_{n+2}, \tau_{k+1} \leqslant \frac{1}{2}\right) \leqslant c 2^{\beta k-\lambda k^{2}} .
$$

Fix $k_{0}$ such that

$$
\sum_{k=k_{0}}^{\infty} k^{2} 2^{-k}<\frac{1}{2}
$$

and sum this estimate for $k_{0} \leqslant k<\infty$ : this leads to

$$
P\left(\forall k \geqslant k_{0}, \tau_{k}-\tau_{k+1} \leqslant k^{2} 2^{-k} \mid E_{n+2}\right) \geqslant 1-c \sum_{k=k_{0}}^{\infty} 2^{\beta k-\lambda k^{2}} .
$$

In particular, if $k_{0}$ is taken large enough, this probability is greater than $1 / 2$, and we obtain

$$
P\left(\tau_{k_{0}} \leqslant \frac{1}{2} \mid E_{n+2}\right) \geqslant \frac{1}{2} .
$$

It is then sufficient to combine this and Eq. (6) to get

$$
P\left(U_{n+2} \mid E_{n+2}\right) \geqslant c 2^{-\beta k_{0}}>0,
$$

and is can be seen that the obtained constant does not depend on the configuration at radius $\mathrm{e}^{n}$ - provided $E_{n}$ is satisfied. 
The first consequence of the end-separation lemma is $P\left(E_{n}, U_{n}\right) \asymp q_{n}$; but it is easy to see, using estimates on Brownian motion in wedges again and the strong Markov property, that

$$
P\left(E_{n+1} \mid E_{n}, U_{n}\right) \geqslant c>0
$$

(with $c$ independent of $n$ ), and combining both estimates leads to $q_{n+1} \geqslant c q_{n}$, i.e. $q_{n+1} \asymp q_{n}$. Now if $\bar{q}_{n}$ stands for the upper bound for the non-intersection probabilities, namely

$$
\bar{q}_{n} \triangleq \operatorname{Sup}_{B_{0}^{1}, B_{0}^{2} \in \mathbb{U}} P\left(E_{n} \mid B_{0}^{1}, B_{0}^{2}\right),
$$

the previous remark concerning the law of $W_{\tau_{1}(W)}$ can be used to prove that $\bar{q}_{n} \leqslant c q_{n-1}$ : hence,

$$
\bar{q}_{n} \asymp q_{n} .
$$

Now that we know that paths conditioned not to intersect have a good chance to exit a disk at a large distance from each other, what remains to be proved is that paths starting from distant points on $\mathcal{C}\left(0, \mathrm{e}^{n}\right)$ remain well separated for a sufficiently long time and become (in a sense to be specified later) independent from their behaviour before radius $\mathrm{e}^{n}$.

Lemma (Start-separation). - Let $\alpha$ and $\eta$ be as in the technical lemma, $\eta^{\prime}=\eta / 2$ and $\alpha^{\prime}=(2 \pi+\alpha) / 2 ;$ introduce

$$
\begin{aligned}
& J_{n}^{1}=\left\{B_{\left[0, \tau_{n}^{1}\right]}^{1} \cap \mathcal{B}(0,2) \subset-W_{2 \pi-\alpha^{\prime}-\eta^{\prime}} \backslash \mathcal{B}\left(0,1-\eta^{\prime}\right)\right\}, \\
& J_{n}^{2}=\left\{A B_{\left[0, \tau_{n}^{2}\right]}^{2} \cap \mathcal{B}(0,2) \subset W_{\alpha^{\prime}} \backslash \mathcal{B}\left(0,1-\eta^{\prime}\right)\right\},
\end{aligned}
$$

and $\widetilde{E}_{n}=E_{n} \cap J_{n}^{1} \cap J_{n}^{2}$. Define $\tilde{q}_{n}$ as

$$
\tilde{q}_{n}(x, y)=P\left(\widetilde{E}_{n} \mid B_{0}^{1}=x, B_{0}^{2}=y\right) .
$$

Then there exists $c>0$ such that, for all $n \geqslant 2$ and uniformly on all pairs $(x, y)$ satisfying $U_{0}$ (i.e., both having modulus 1 and such that $U_{0}$ holds when $B_{0}^{1}=x$ and $B_{0}^{2}=y$ ):

$$
\tilde{q}_{n}(x, y) \geqslant c q_{n} .
$$

Proof. - Introduce the following ("forbidden") sets:

$$
\begin{aligned}
& K^{1}=\left(\mathcal{B}(0,2) \backslash-W_{2 \pi-\alpha^{\prime}-\eta^{\prime}}\right) \cup \mathcal{B}\left(0,1-\eta^{\prime}\right) ; \\
& K^{2}=\left(\mathcal{B}(0,2) \backslash W_{\alpha^{\prime}}\right) \cup \mathcal{B}\left(0,1-\eta^{\prime}\right) .
\end{aligned}
$$

For all $n$ we have $J_{n}^{1}=\left\{B_{\left[0, \tau_{n}^{1}\right]}^{1} \cap K^{1}=\emptyset\right\}$ and $J_{n}^{2}=\left\{A B_{\left[0, \tau_{n}^{2}\right]}^{2} \cap K^{2}=\emptyset\right\}$. For the rest of the proof we shall fix $n$, and condition the paths by their starting points; introduce the following stopping times (for positive values of $k$ ):

$$
\begin{aligned}
& T_{0}^{1}=\operatorname{Inf}\left\{t>0: B_{[0, t]}^{1} \cap \mathcal{C}(0,3) \neq \emptyset\right\}, \\
& S_{k}^{1}=\operatorname{Inf}\left\{t>T_{k-1}^{1}: B_{\left[T_{k-1}^{1}, t\right]}^{1} \cap K^{1} \neq \emptyset\right\}, \\
& T_{k}^{1}=\operatorname{Inf}\left\{t>S_{k}^{1}: B_{\left[S_{k}^{1}, t\right]}^{1} \cap \mathcal{C}(0,3) \neq \emptyset\right\},
\end{aligned}
$$


and $S_{k}^{2}, T_{k}^{2}$ similarly, replacing all occurrences of $B^{1}$ by $A B^{2}$ and $K^{1}$ by $K^{2}$. We shall also use the notation $N^{i}$ for the number of crossings by $B^{1}$ (resp. $A B^{2}$ ) between $K^{i}$ and $\mathcal{C}(0,3)$, defined as

$$
N^{i} \triangleq \operatorname{Max}\left\{k: S_{k}^{i}<\tau_{n}^{i}\right\} .
$$

With those notations, $J_{n}^{i}=J_{1}^{i} \cap\left\{N^{i}=0\right\}$ and a.s. $N^{i}<\infty$. Moreover, uniformly on the starting points considered here (satisfying the condition $U_{0}$ ), we have $P\left(J_{1}^{i}\right) \geqslant c>0$ by the technical lemma, where $c$ depends only on $\eta$.

First, we split the event $E_{n}$ according to the value of, say, $N^{2}$ : we write $P\left(E_{n}\right)=$ $\sum_{k=0}^{\infty} P\left(E_{n}, N^{2}=k\right)$. By the Beurling estimate, on $\left\{N^{2} \geqslant k\right\}$, the probability that $B_{\left[0, \tau_{n}^{1}\right]}^{1}$ and $A B_{\left[S_{k}^{2}, T_{k}^{2}\right]}^{2}$ do not intersect is bounded by some universal constant $\lambda<1$ (which can even be chosen independent of $A$ ), independently of $B^{1}$ and the two remaining parts of $B^{2}$. By the strong Markov property at time $T_{k}^{2}$, when $N^{2}=k$ the probability that $A B^{2}$ after $T_{k}^{2}$ does not intersect $B^{1}$ is bounded by $P\left(B^{1} \cap A B_{\left[T_{0}^{2}, \tau_{n}^{2}\right]}^{2}=\emptyset, N^{2}=0\right)$ (i.e., the path after $T_{k}^{2}$ when $N^{2}=k$ is the same as the entire path when $N^{2}=0$ ). Introducing those two estimates in the sum leads to

$$
P\left(E_{n}\right) \leqslant \sum_{k=0}^{\infty} \lambda^{k} P\left(E_{n}, N^{2}=0\right)=\frac{1}{1-\lambda} P\left(E_{n}, N^{2}=0\right) .
$$

Doing this decomposition again according to $N^{1}$ (with the same constant $\lambda<1$ ) we then obtain

$$
P\left(E_{n}\right) \leqslant \frac{1}{(1-\lambda)^{2}} P\left(E_{n}, N^{1}=N^{2}=0\right),
$$

i.e. $P\left(N^{1}=N^{2}=0 \mid E_{n}\right) \geqslant(1-\lambda)^{2}>0$. This, and the previous remark that $P\left(J_{n}^{i} \mid N^{i}=\right.$ 0 ) is bounded below by a constant provided that the starting points satisfy $U_{0}$, gives:

$$
P\left(\widetilde{E}_{n} \mid B_{0}^{1}=x, B_{0}^{2}=y\right) \geqslant c P\left(E_{n} \mid B_{0}^{1}=x, B_{0}^{2}=y\right) .
$$

Conditioning on $B^{2}$ shows that the map

$$
f: x \mapsto P\left(E_{n} \mid B_{0}^{1}=x, B_{0}^{2}=1\right)
$$

is harmonic and does not vanish on the complement of $\bar{A}$. Moreover, its supremum on the unit circle is equal to $\bar{q}_{n}$ by definition: Applying the Harnack principle then proves that $f$ is bounded below by $c q_{n}$ on the set of $x$ satisfying $U_{0}$, which completes the proof.

Another estimate can be obtained using the very same proof: Only keeping the conditions involving disks and relaxing those involving wedges, we obtain

$$
P\left(B_{\left[0, \tau_{n}^{1}\right]}^{1} \cap B(0,1-\eta)=\emptyset, A B_{\left[0, \tau_{n}^{2}\right]}^{2} \cap \mathcal{B}(0,1-\eta)=\emptyset \mid B_{0}^{1}, B_{0}^{2}, E_{n}\right) \geqslant c>0,
$$

where $c$ does not depend on the initial positions $B_{0}^{1}$ and $B_{0}^{2}$, nor on $n$ (it clearly depends on $\eta$, though, and a closer look at the proof shows that we can ensure $c>\eta^{\beta}$ as $\eta \rightarrow 0$, 
for some $\beta>0$ ). This estimate will be needed in the derivation of Hausdorff dimensions, cf. Section 3.

We now have all the needed estimates to derive the lower bound in the sub-additivity condition, and hence the conclusion of the theorem. Take two paths with independent starting points uniformly distributed on the unit circle and killed at radius $\mathrm{e}^{m+n}$, conditioned not to intersect between radii 1 and $\mathrm{e}^{n}$. This happens with probability $q_{n}$. With large probability (i.e., with a positive probability, independent of $m$ and $n$ ) the paths up to radius $\mathrm{e}^{n}$ end up "well separated" in the sense of the end-separation lemma. In particular, the points where they reach radius $\mathrm{e}^{n}$, after suitable rescaling, satisfy the hypothesis of the start-separation lemma: Hence with probability greater that $c q_{m}$, the paths between radii $\mathrm{e}^{n}$ and $\mathrm{e}^{m+n}$ remain separated up to radius $\mathrm{e}^{n+1}$, do not reach radius $(1-\eta) \mathrm{e}^{n}$ anymore and do not intersect up to radius $\mathrm{e}^{m+n}$. Under those conditions, it is easy to see that the paths do not meet at all. So $q_{m+n} \geqslant c q_{m} q_{n}$ for some positive $c$, and we get the conclusion.

Some adaptations are needed if $A$ is included in an annulus, say $\{r<|z|<R\}$ with $r<1<R$. First, replace all occurrences of $e$ by $e_{0}$, with $e_{0}$ chosen larger than $10 R / r$, and in the start-separation lemma, replace $\mathcal{B}(0,1-\eta)$ by $\mathcal{B}(0, r / 2 R)$ in the definition of the $J_{n}$. As long as $r$ and $R$ are fixed, this changes nothing to the proof, except that the constants we obtain will then depend on $R / r$ - which itself is bounded provided $A$ remains a subset of some fixed nice set.

A more serious problem arises if the complement of $\bar{A}$ is not connected (i.e., if $A$ has holes), since the natural domain of the function $f$ (as defined by Eq. (11)) is itself not connected. However, since $A$ is nice, its complement has exactly one unbounded component, and it is easy to see that if $x$ is not in this component then $f(x)$ vanishes for $n \geqslant 1$. Hence, nothing changes (as far as non-intersection properties are concerned) when $A$ is replaced by the complement of the infinite component of its complement (i.e. when filling the holes in $A$ ).

In fact, a stronger result can be derived: If the starting points $B_{0}^{1}$ and $B_{0}^{2}$ are fixed, then $P\left(E_{n} \mid B_{0}^{1}, B_{0}^{2}\right)$ is equivalent to $c \mathrm{e}^{-n \xi(A)}$, where $c$ is a function of $B_{0}^{1}$ and $B_{0}^{2}$ satisfying $c \leqslant c_{0} d\left(B_{0}^{1}, A B_{0}^{2}\right)^{\beta}$. This estimate is related to a strong convergence result on the law of paths conditioned by $B^{1} \cap A B^{2}=\emptyset$. However, proving this result would be much more involved (cf. [18] for the proof in the case $A=\{1\}$ ).

\section{Properties of the function $A \mapsto \xi(A)$}

We first list a few simple properties of the function $A \mapsto \xi(A)$. For $p \in \mathbb{Z}$ and $A \subset \mathbb{C}$, introduce $A^{p}=\left\{z^{p}, z \in A\right\}$ and let $A^{*}=\{\bar{z}, z \in A\}$.

PROPOSITION 1. - Is these statements, all sets are assumed to be non-empty but do not need to be nice:

(i) $\xi$ is non-decreasing: if $A \subset A^{\prime}$ then $\xi(A) \leqslant \xi\left(A^{\prime}\right)$;

(ii) $\xi$ is homogeneous: if $\lambda \in \mathbb{C}^{*}$ then $\xi(\lambda A)=\xi(A)$;

(iii) $\xi$ is symmetric: $\xi\left(A^{-1}\right)=\xi\left(A^{*}\right)=\xi(A)$;

(iv) $\xi$ has the following property: if $n \geqslant 1$ then

$$
\xi\left(\bigcup \mathrm{e}^{2 \mathrm{i} k \pi / n} A\right)=n \xi\left(A^{n}\right) .
$$


Proof. - (i) This is a trivial consequence of $p_{R}(A) \geqslant p_{R}\left(A^{\prime}\right)$.

(ii) Applying the scaling property with factor $|\lambda|$ to $B^{2}$ proves that one can suppose $|\lambda|=1$; in which case we have $p_{R}(A)=p_{R}(\lambda A)$ (because the starting points are uniformly distributed on the unit circle).

(iii) Simply exchange $B^{1}$ and $B^{2}$ for $A^{-1}$, and say that the complex conjugate of a Brownian path is still a Brownian path to get $A^{*}$.

(iv) This is a consequence of the analyticity of the mapping $z \mapsto z^{n}$ (hence the fact that $\left(\left(W_{t}\right)^{n}\right)$ is a Brownian path if $W$ is one) together with the remark that the existence of $s, t>0$ and $z \in A^{n}$ with $\left(B_{s}^{1}\right)^{n}=z\left(B_{t}^{2}\right)^{n}$ is equivalent to the existence of $z^{\prime}$ in $\bigcup \mathrm{e}^{2 i k \pi / n} A$ with $B_{t}^{1}=z^{\prime} B_{t}^{2}$ - note that the mapping also has an influence on $R$, hence the factor $n$.

We now turn our attention toward regularity properties of the function $A \mapsto \xi(A)$ - the following result being a key step toward the derivation of dimensions in the next section. Introduce the Hausdorff distance between compact subsets of the plane (cf. Appendix A for details). It will be convenient here to define neighbourhoods by $V_{r}(A)=\left\{x \mathrm{e}^{z}, x \in A,|z|<r\right\}$ instead of the usual $A+\mathcal{B}(0, r)$ - leading to the logarithmic Hausdorff distance. The (logarithmic) Hausdorff topology is the metric topology derived from this distance.

PROPOSITION 2. - $\xi$ is continuous on the collection of nice sets, endowed with the logarithmic Hausdorff topology. For any nice set $A_{0}, \xi$ is uniformly continuous in $\left\{A: A \subset A_{0}\right\}$.

Proof. - The proof relies on the uniformity of the strong approximation in Theorem 1: fix a nice set $A_{0}$ and assume all sets considered here are subsets of $A_{0}$. The constants $c, c_{-}$and $c_{+}$appearing during the proof may only depend on $A_{0}$.

First, fix $R>1$ and condition all events by $B_{\left[0, T_{R+1}^{2}\right]}^{2}-$ i.e., fix the second path. For all $A \subset A_{0}$, let

$$
\mathrm{d}_{R}(A)=\mathrm{d}_{H}\left(B_{\left[0, T_{R}^{1}\right]}^{1}, A B_{\left[0, T_{R}^{2}\right]}^{2}\right) ;
$$

for all $\varepsilon>0$ introduce the stopping time

$$
S_{\varepsilon}=\operatorname{Inf}\left\{t: \mathrm{d}_{H}\left(B_{t}^{1}, A B_{\left[0, T_{R}^{2}\right]}^{2}\right)<\varepsilon\right\} .
$$

Note that $\left\{\mathrm{d}_{R}(A)<\varepsilon\right\}=\left\{S_{\varepsilon}<T_{R}^{1}\right\}$. On this event, the strong Markov property shows that $B_{S_{\varepsilon}+\text {. }}^{1}$ is a Brownian path starting $\varepsilon$-close to $A B^{2}$. By Beurling's theorem, the probability that they do not meet before radius $R+1$ is smaller than the corresponding probability for a path near a half line; hence,

$$
P\left(B_{\left[S_{\varepsilon}, T_{R+1}^{1}\right]}^{1} \cap A B_{\left[0, T_{R+1}^{2}\right]}^{2}=\emptyset \mid \mathrm{d}_{R}(A)<\varepsilon\right) \leqslant \sqrt{\varepsilon},
$$

so that, considering the whole path, $P\left(E_{R+1} \mid \mathrm{d}_{R}(A)<\varepsilon\right) \leqslant \sqrt{\varepsilon}$. Apply the Bayes formula:

$$
P\left(\mathrm{~d}_{R}(A)<\varepsilon \mid E_{R+1}\right)=\frac{P\left(\mathrm{~d}_{R}(A)<\varepsilon\right)}{P\left(E_{R+1}\right)} P\left(E_{R+1} \mid \mathrm{d}_{R}(A)<\varepsilon\right) ;
$$


since we know that $P\left(E_{R+1}\right) \geqslant c_{-}(R+1)^{-\xi(A)}$ with $\xi(A) \leqslant \xi\left(A_{0}\right)$ we finally obtain

$$
P\left(\mathrm{~d}_{R}(A)<\varepsilon \mid E_{R+1}\right) \leqslant c R^{\xi\left(A_{0}\right)} \sqrt{\varepsilon} .
$$

From now on, we shall assume that $\varepsilon$ is sufficiently small to make the obtained bound smaller that 1 . Taking the complement leads to

$$
P\left(\mathrm{~d}_{R}(A) \geqslant \varepsilon \mid E_{R+1}\right) \geqslant 1-c R^{\xi\left(A_{0}\right)} \sqrt{\varepsilon} .
$$

Now, remark that when $\mathrm{d}_{R}(A) \geqslant \varepsilon$ and $\mathrm{d}_{H}\left(A, A^{\prime}\right)<\varepsilon / R$, we have $B_{\left[0, T_{R}^{1}\right]}^{1} \cap A^{\prime} B_{\left[0, T_{R}^{2}\right]}^{2}=$ $\emptyset$ : from this and the previous equation it follows that, as long as $A$ and $A^{\prime}$ remain subsets of $A_{0}$,

$$
\mathrm{d}_{H}\left(A, A^{\prime}\right)<\frac{\varepsilon}{R} \quad \Rightarrow \quad p_{R}\left(A^{\prime}\right) \geqslant\left(1-c R^{\xi\left(A_{0}\right)} \sqrt{\varepsilon}\right) p_{R+1}(A) .
$$

We can apply the estimates on $p_{R}$ we derived in Theorem $1-$ i.e., $p_{R}(A) \asymp$ $p_{R+1}(A) \asymp R^{-\xi(A)}$ : still for $\mathrm{d}_{H}\left(A, A^{\prime}\right)<\varepsilon / R$ and $A, A^{\prime}$ inside $A_{0}$ we get

$$
c_{+} R^{-\xi\left(A^{\prime}\right)} \geqslant\left(1-c R^{\xi\left(A_{0}\right)} \sqrt{\varepsilon}\right) c_{-} R^{-\xi(A)},
$$

and taking the logarithm of each side of the inequality leads to

$$
\log c_{+}-\xi\left(A^{\prime}\right) \log R \geqslant \log c_{-}+\log \left(1-c R^{\xi\left(A_{0}\right)} \sqrt{\varepsilon}\right)-\xi(A) \log R,
$$

hence after suitable transformations:

$$
\xi\left(A^{\prime}\right) \leqslant \xi(A)+\frac{c}{\log R}-\frac{\log \left(1-c R^{\xi\left(A_{0}\right)} \sqrt{\varepsilon}\right)}{\log R} .
$$

Fix $\eta>0$, and choose $R$ such that $c / \log R<\eta / 2$. It is then possible to take $\varepsilon$ sufficiently small so that $\left|\log \left(1-c R^{\xi\left(A_{0}\right)} \sqrt{\varepsilon}\right)\right|<(\eta \log R) / 2$; for $\mathrm{d}_{H}\left(A, A^{\prime}\right)<\varepsilon / R$ we then have $\xi\left(A^{\prime}\right) \leqslant \xi(A)+\eta$, hence by symmetry $\left|\xi\left(A^{\prime}\right)-\xi(A)\right| \leqslant \eta$. This proves that $\xi$ is uniformly continuous on $\mathcal{P}_{c}\left(A_{0}\right)$, for all $A_{0}$, hence continuous on the family of nice sets.

Remark 1. - Eq. (13) allows the derivation of an explicit modulus of continuity for $\xi$ inside $A_{0}$, of the form

$$
\left|\xi\left(A^{\prime}\right)-\xi(A)\right| \leqslant \frac{C\left(A_{0}\right)}{\left|\log \mathrm{d}_{H}\left(A, A^{\prime}\right)\right|}
$$

(take $\left.R=d^{-1 / 2 \xi\left(A_{0}\right)}\right)$. But since $C\left(A_{0}\right)$ is not known, this does not provide numerical bounds for $\xi$.

Remark 2. - Inside a nice set, the usual and logarithmic Hausdorff topologies are equivalent, so the introduction of "exponential neighbourhoods" in Proposition 2 can seem artificial; however, it leads to constants that do not vary when $A$ is multiplied by some constant (as in Proposition 1, point (ii)), hence uniform continuity holds on the 
collection of nice sets contained in a fixed wedge and in some annulus $\{r<|z|<c r\}$ for fixed $c$-which is wrong for the usual Hausdorff topology, as a consequence of the homogeneity of $\xi$ applied for small $|\lambda|$.

Note that uniform continuity cannot hold on the family of nice sets contained in a given annulus since $\xi$ would then be bounded (by a compactness argument), which it is not: the exponent associated to a circle is infinite.

\section{Hausdorff dimension of the corresponding subsets of the path}

\subsection{Conformally invariant subsets of the Brownian path}

It is well known that the Brownian path is invariant in law under conformal transformations; in this section, we study subsets of the Brownian curve that are also invariant under conformal maps. A first example is the set of so-called Brownian cutpoints, i.e. points $B_{t}$ such that $B_{[0, t)}$ and $B_{(t, 1]}$ are disjoint; these points form a set of Hausdorff dimension $2-\xi(\{1\})=3 / 4$. Related to those are local cut-points, i.e. points such that there exists $\varepsilon>0$ satisfying $B_{[t-\varepsilon, t)} \cap B_{(t, t+\varepsilon]}=\emptyset$ - the dimension is the same as for global cut-points. Other examples are given by Lawler in [11]: in particular the set of pioneer points (such that $B_{t}$ lies on the frontier of the infinite component of the complement of $B_{[0, t]}$ ), related to the disconnection exponent $\eta_{1}$; frontier points (points of the boundary of the infinite component of the complement of $\left.B_{[0,1]}\right)$, related to the disconnection exponent for two paths in the plane. Another exceptional subset of the path is the set of cone points (such that $B_{[0, t]}$ is contained in a cone of endpoint $B_{t}$ ), related to the cone exponents (studied in [19] for example).

We will use the exponents introduced in the previous sections to describe a family of exceptional sets, indexed by a subset $A$ of the complex plane, having dimension $2-\xi(A)$, and that are invariant under conformal transformations, as follows. Fix a Brownian path $B_{[0,1]}$, a subset $A$ of the complex plane, and introduce the following times for all $t \in(0,1)$ and $r>0$ :

$$
T_{r}(t)=\operatorname{Inf}\left\{s>t:\left|B_{s}-B_{t}\right|=r\right\}, \quad S_{r}(t)=\operatorname{Sup}\left\{s<t:\left|B_{s}-B_{t}\right|=r\right\} .
$$

Definition. - If $0<\varepsilon<R$ and $t \in(0,1)$, let

$$
Z_{t}^{[\varepsilon, R]}(B)=\left\{\frac{B_{s}-B_{t}}{B_{s^{\prime}}-B_{t}}: s \in\left[T_{\varepsilon}(t), T_{R}(t)\right], s^{\prime} \in\left[S_{R}(t), S_{\varepsilon}(t)\right]\right\}
$$

and introduce $\mathcal{E}_{A}^{[\varepsilon, R]}=\left\{B_{t}: Z_{t}^{[\varepsilon, R]} \cap A=\emptyset\right\}$. Then, letting $\varepsilon$ go to 0 :

$$
Z_{t}^{R}=\bigcup_{\varepsilon>0} Z_{t}^{[\varepsilon, R]}, \quad Z_{t}=\bigcap_{R>0} Z_{t}^{R}, \quad \widetilde{Z}_{t}=\bigcap_{R>0} \overline{Z_{t}^{R}} ;
$$

define $\mathcal{E}_{A}^{R}, \mathcal{E}_{A}$ and $\widetilde{\mathcal{E}}_{A}$ accordingly.

We shall also use the notation $\mathcal{T}_{A}=\left\{t: B_{t} \in \mathcal{E}_{A}\right\}$, for the set of $A$-exceptional times, and $\widetilde{\mathcal{T}}_{A}=\left\{t: B_{t} \in \widetilde{\mathcal{E}}_{A}\right\}$, for the set of $A$-strongly exceptional times. 
Note that, since 0 is polar for planar Brownian motion, $Z$ is well-defined for almost any $t$. For $A=\{1\}, \mathcal{E}_{A}$ is the set of local cut-points; more generally, $B_{t}$ is in $\mathcal{E}_{A}$ if, and only if, for some $\varepsilon>0$, we have

$$
\left(B_{(t, t+\varepsilon]}-B_{t}\right) \cap A\left(B_{[t-\varepsilon, t)}-B_{t}\right)=\emptyset,
$$

so the setup looks similar to the definition of the exponent $\xi(A)$. It is easy to see that for all fixed $t>0$, a.s. $Z_{t}=\mathbb{C}^{*}$ and $\widetilde{Z}_{t}=\mathbb{C}$, so that for $A \neq \emptyset, P\left(t \in \mathcal{T}_{A}\right)=0$, leading to $E\left(\mu\left(\mathcal{T}_{A}\right)\right)=0$, i.e. $\mu\left(\mathcal{T}_{A}\right)=0$ almost surely - hence the term "exceptional points".

The set $\mathcal{E}_{A}$ of A-exceptional points is generally not conformally invariant. However, it is the case for strongly exceptional points:

PROPOSITION 3. - Let $\Phi$ be a conformal map on a neighbourhood $\Omega$ of 0 , with $\Phi(0)=0$, and let $B^{\Omega}$ be $B$ stopped at its first hitting of $\partial \Omega$. By conformal invariance of planar Brownian motion, $\Phi\left(B^{\Omega}\right)$ is a Brownian path stopped at its first hitting of $\partial \Phi(\Omega)$. Moreover, we have

$$
\widetilde{\mathcal{E}}_{A}\left(\Phi\left(B^{\Omega}\right)\right)=\Phi\left(\widetilde{\mathcal{E}}_{A}\left(B^{\Omega}\right)\right) .
$$

Proof.-We prove that $\widetilde{Z}$ is invariant. It is sufficient to prove the following characterization:

$$
z \in \widetilde{Z}_{t}(B) \quad \Longleftrightarrow \quad \exists\left(s_{n}\right) \downarrow 0,\left(s_{n}^{\prime}\right) \downarrow 0: \frac{B_{t+s_{n}}-B_{t}}{B_{t-s_{n}^{\prime}}-B_{t}} \rightarrow z,
$$

as conformal maps conserve the limits of such quotients. Such a sequence is easily constructed using the very definition of $\widetilde{Z}$.

Note that nothing in the preceding uses the fact that $B$ be a Brownian path, except for the remark about $P\left(t \in \mathcal{T}_{A}\right)$. The remaining of the present section is dedicated to deriving the Hausdorff dimension of $\mathcal{E}_{A}$ and $\widetilde{\mathcal{E}}_{A}$. It will be more convenient to work in the time set, so introduce

$$
\mathcal{T}_{A}^{[\varepsilon, R]}=\left\{t \in[0,1]:\left(B_{[t-R, t-\varepsilon]}-B_{t}\right) \cap A\left(B_{[t+\varepsilon, t+R]}-B_{t}\right)=\emptyset\right\} .
$$

The scaling property of Brownian motion can then be used to show, as in $[9$, Lemmas 3.14-3.16], that Theorem 1 implies the following, provided $A$ is nice:

$$
P\left(t \in \mathcal{T}_{A}^{[\varepsilon, R]}\right) \asymp\left(\frac{\varepsilon}{R}\right)^{\xi(A) / 2} .
$$

\subsection{Second moments}

Fix $R>0$. The purpose of this subsection is to give an estimate of the probability that two times $t$ and $t^{\prime}$ are $A$-exceptional times, i.e. are both in $\mathcal{T}_{A}^{[\varepsilon, R]}$. To get an upper bound on this probability, the idea will be to dissociate the microscopic and macroscopic 
scales, giving respectively the first and second factor in the following estimate:

$$
P\left(t, t^{\prime} \in \mathcal{T}_{A}^{[\varepsilon, R]}\right) \leqslant c\left[\frac{\varepsilon}{R}\right]^{\xi(A)}\left[1 \vee\left|t-t^{\prime}\right|^{-\xi(A) / 2}\right] .
$$

If $t<t^{\prime}$ are two times, introduce the "mesoscopic" scale $d=\left|t^{\prime}-t\right|$, and separate the following three cases:

- If $d>2 R$ (long-range interaction), the events $E_{t} \wedge\left\{t \in \mathcal{T}_{A}^{[\varepsilon, R]}\right\}$ and $E_{t^{\prime}}$ are independent, leading to the right second-order moment;

- If $R / 2 \leqslant d \leqslant 2 R$ (medium-range interaction), the trivial bound $P\left(E_{x}, E_{y}\right) \leqslant$ $C(2 \varepsilon / d)^{\xi(A)}$ (obtained by forgetting what happens after radius $d / 2$ ) gives the needed contribution.

- If $d<R / 2$ (short-range interaction), a little more work is required. Introduce the following times:

$$
\begin{aligned}
& \widetilde{T}_{r}(x)=\operatorname{Min}\left(x+r, \operatorname{Inf}\left\{x^{\prime}>x:\left|B_{x}-B_{x^{\prime}}\right|=r^{1 / 2}\right\}\right), \\
& \widetilde{S}_{r}(x)=\operatorname{Max}\left(x-r, \operatorname{Sup}\left\{x^{\prime}<x:\left|B_{x}-B_{x^{\prime}}\right|=r^{1 / 2}\right\}\right) .
\end{aligned}
$$

First, $E_{t}$ and $E_{t^{\prime}}$ imply two independent events:

$$
\begin{aligned}
& E_{1}:\left(B_{\left[t+\varepsilon, \widetilde{T}_{d / 2}(t)\right]}-B_{t}\right) \cap A\left(B_{\left[\widetilde{S}_{d / 2}(t), t-\varepsilon\right]}-B_{t}\right)=\emptyset, \\
& E_{2}:\left(B_{\left[t^{\prime}+\varepsilon, \widetilde{T}_{d / 2}\left(t^{\prime}\right)\right]}-B_{t}^{\prime}\right) \cap A\left(B_{\left[\widetilde{S}_{d / 2}\left(t^{\prime}\right), t^{\prime}-\varepsilon\right]}-B_{t}^{\prime}\right)=\emptyset ;
\end{aligned}
$$

as in [9], it can be proved that $P\left(E_{1}\right) \asymp P\left(t \in \mathcal{T}_{A}^{[\varepsilon, d / 2]}\right) \asymp(\varepsilon / d)^{\xi(A) / 2}$. Let

$$
\delta=\operatorname{Max}\left((d / 2)^{1 / 2},\left|B_{\widetilde{T}_{d / 2}\left(t^{\prime}\right)}\right|\right) .
$$

$\delta$ is stochastically dominated by the sum of $(d / 2)^{1 / 2}$ and a Gaussian variable $\mathcal{N}(0, d)$ (accounting for the behaviour of $B$ between the times $\widetilde{T}_{d / 2}(t)$ and $\widetilde{S}_{d / 2}\left(t^{\prime}\right)$ ). Moreover, conditionally to the value of $\delta$, the joint distribution of $B$ at times $S_{2 \delta}(t)$ and

$$
T^{\prime} \triangleq \operatorname{Inf}\left\{x>t^{\prime}:\left|B_{x}-t\right|=2 \delta\right\}
$$

is absolutely continuous with respect to the Lebesgue measure on $\mathcal{C}(0,2 \delta)^{2}$, and its density is bounded above and below by absolute constants. Lastly, $E_{t}$ and $E_{t^{\prime}}$ imply that

$$
\left(B_{\left[T^{\prime}, t+R\right]}-B_{t}\right) \cap A\left(B_{\left[t-R, S_{2 \delta}(t)\right]}-B_{t}\right)=\emptyset,
$$

and (still conditionally on $\delta$ ) the probability of this event is bounded above by $C(2 \delta)^{\xi(A)}$ by Theorem 1 . But the previous remark on the law of $\delta$ shows that

$$
E\left(\delta^{\xi(A)}\right) \leqslant C d^{\xi(A) / 2}
$$

hence finally the correct estimate:

$$
P\left(E_{t}, E_{t^{\prime}}\right) \leqslant C\left(\frac{\varepsilon}{d}\right)^{\xi(A)} d^{\xi(A) / 2}=C \frac{\varepsilon^{\xi(A)}}{\left|t-t^{\prime}\right|^{\xi(A) / 2}} .
$$


So in the case of exceptional points defined locally, bounds on second moments are not difficult to derive (and this "scale separation" construction can be used in various setups). In contrast, if the whole path was to influence every single point, interactions would not be that easy to classify.

\subsection{Hausdorff dimensions}

The main result of this section is the following:

THEOREM 2. - Let $\left(B_{t}\right)_{t \in[0,1]}$ be a planar Brownian path. If $A$ is any nice subset of the complex plane such that $\xi(A) \leqslant 2$, then almost surely

$$
\operatorname{dim}_{H}\left(\mathcal{E}_{A}(B)\right)=\operatorname{dim}_{H}\left(\widetilde{\mathcal{E}}_{A}(B)\right)=2-\xi(A) .
$$

In particular, both subsets are a.s. non-empty and dense in the path if $\xi(A)<2$. If $\xi(A)>2, \mathcal{E}_{A}(B)=\widetilde{\mathcal{E}}_{A}(B)=\emptyset$ almost surely.

Proof. - The first step in the proof is the statement of a zero-one law:

Lemma 1. - The dimension of the set of all A-exceptional points (resp. of A-strong exceptional points) has an almost sure value. More precisely, there exist $\delta_{A}$ and $\tilde{\delta}_{A}$ in $[0,2]$ such that

$$
P\left(\operatorname{dim}_{H}\left(\mathcal{E}_{A}\right)=\delta_{A}\right)=P\left(\operatorname{dim}_{H}\left(\widetilde{\mathcal{E}}_{A}\right)=\tilde{\delta}_{A}\right)=1 .
$$

Moreover, the following holds with probability 1 (and the same for $\widetilde{\mathcal{E}}_{A}$ also):

$$
\forall s<t \quad \operatorname{dim}_{H}\left(\mathcal{E}_{A}\left(B_{[s, t]}\right)\right)=\delta_{A} .
$$

Proof. - The proof is the same in both cases; we perform it here for $\delta_{A}$. Introduce the following random variables in $[0,2]$ :

$$
Z=\operatorname{dim}_{H}\left(\mathcal{E}_{A}\right), \quad Z_{-}=\operatorname{dim}_{H}\left(\mathcal{E}_{A}\left(B_{[0,1 / 3]}\right)\right), \quad Z_{+}=\operatorname{dim}_{H}\left(\mathcal{E}_{A}\left(B_{[2 / 3,1]}\right)\right) .
$$

The scaling property, associated with the Markov property, shows that these three variables have the same law; basic properties of the Hausdorff dimension imply that $Z \geqslant Z_{-} \vee Z_{+}$; and locality proves that $Z_{-}$and $Z_{+}$are independent.

$0 \leqslant Z_{-} \leqslant Z \leqslant 2$ with the same mean value: from here follows that $P\left(Z_{-}=Z\right)=1$. By the same argument $P\left(Z_{+}=Z\right)=1$, hence $P\left(Z_{-}=Z_{+}\right)=1 ; Z_{-}$and $Z_{+}$being independent, this is only possible if they are deterministic: thus giving the existence of $\delta_{A}$ as their common almost sure value.

Now if $0 \leqslant s<t \leqslant 1$ the dimension of $\mathcal{E}_{A}\left(B_{[s, t]}\right)$ is (almost surely) $\delta_{A}$. This holds at the same time for all rational $s, t$; then it suffices to note that $\operatorname{dim}_{H}\left(\mathcal{E}_{A}\left(B_{I}\right)\right)$ is increasing in $I$ to extend the equality to all $s<t$.

From this lemma follows that as soon as $\mathcal{E}_{A}$ has positive dimension it is dense in the path.

For convenience we will prove the result in the time set, i.e. we shall compute the dimension of $\mathcal{T}_{A}$; it is known that planar Brownian motion doubles Hausdorff dimensions 
(i.e., with probability 1 , for any Borel subset $I$ of $[0,1], \operatorname{dim}_{H}\left(B_{I}\right)=2 \operatorname{dim}_{H}(I)-$ cf. [7]), whence $\operatorname{dim}_{H}\left(\mathcal{E}_{A}\right)=2 \operatorname{dim}_{H}\left(\mathcal{T}_{A}\right)$. Moreover, to avoid problems near 0 and 1 we shall suppose that $B$ is defined for $t \in \mathbb{R}$ - this will not change $\mathcal{T}_{A}$ since the definition is local.

First step: lower bound. Fix $R>0$ and let $A_{n}$ be the following set:

$$
A_{n}=\left\{t:\left(B_{\left[t-R, t-2^{-n}\right]}-B_{t}\right) \cap A\left(B_{\left[t+2^{-n}, t+R\right]}-B_{t}\right)=\emptyset\right\} .
$$

For shorter notations, let $s=\xi(A) / 2$; moreover, assume from now on that $s \in(0,1)$ (if $s \geqslant 1$ there is nothing to prove, and since $A \neq \emptyset$ we have $s>0$ anyway). From the previous estimates for first- and second-moments, we obtain

$$
E\left(\mathbb{1}_{A_{n}}(x)\right) \asymp 2^{-s n}, \quad E\left(\mathbb{1}_{A_{n}}(x) \mathbb{1}_{A_{n}}(y)\right) \leqslant c 2^{-2 s n}\left[1 \vee \frac{1}{|y-x|^{s}}\right] .
$$

Introduce the (random) measure $\mu_{n}$ having density $2^{s n} \mathbb{1}_{A_{n}}$ with respect to the Lebesgue measure. It is not hard to derive the following estimates:

$$
\begin{aligned}
E\left(\left\|\mu_{n}\right\|\right) & =\int_{[0,1]} 2^{s n} E\left(\mathbb{1}_{A_{n}}(x)\right) \mathrm{d} x \asymp 1 \\
E\left(\left\|\mu_{n}\right\|^{2}\right) & =\iint_{[0,1]^{2}} 2^{2 s n} E\left(\mathbb{1}_{A_{n}}(x) \mathbb{1}_{A_{n}}(y)\right) \mathrm{d} x \mathrm{~d} y \\
& \leqslant c 2^{s n}\left[\int_{0}^{1} \mathrm{~d} x \int_{x}^{x+2^{-n}} \mathrm{~d} y+\int_{0}^{1-2^{-n}} \mathrm{~d} x \int_{x+2^{-n}}^{1} \frac{2^{-s n} \mathrm{~d} y}{(y-x)^{s}}\right] \\
& \leqslant c 2^{(s-1) n}+c \int_{0}^{1-2^{-n}}\left(\frac{(1-x)^{1-s}}{1-s}-\frac{2^{(s-1) n}}{1-s}\right) \mathrm{d} x \\
& \leqslant c+c 2^{(s-1) n}+c 2^{(s-2) n} \leqslant c .
\end{aligned}
$$

Hence, $\left\|\mu_{n}\right\|$ has finite expectation and finite variance, independent of $n$ : there exists $\varepsilon>0$ satisfying $P\left(\left\|\mu_{n}\right\|>\varepsilon\right)>\varepsilon$ for all positive $n$. Consequently, it is possible, with positive probability, to extract a subsequence $\left(\mu_{n_{k}}\right)$ such that, for all $k,\left\|\mu_{n_{k}}\right\| \geqslant \varepsilon$. By a compactness argument, another extraction leads to a converging subsequence, the limit $\mu$ of which satisfies $\|\mu\| \geqslant \varepsilon . \mu$ is supported on the intersection of the $A_{n}$, this intersection is non-empty: hence $P\left(\cap A_{n} \neq \emptyset\right)>0$.

Introduce then the notion of $r$-energy of a measure: if $v$ is some mass measure supported on a metric space $X$, let

$$
\mathcal{E}_{r}(v) \wedge \iint_{X^{2}} \frac{\mathrm{d} \nu(x) \mathrm{d} v(y)}{\mathrm{d}(x, y)^{r}} .
$$

It is known that if $X$ supports a mass measure of finite $r$-energy, then its Hausdorff dimension is not less than $r$ (cf. [6]). Let then $r \in(0,1-s)$ : a calculation analogous to 
the derivation of (16) leads to

$$
E\left(\mathcal{E}_{r}\left(\mu_{n}\right)\right) \leqslant c+c 2^{(r+s-1) n}+c 2^{(r+s-2) n} \leqslant c .
$$

Performing another subsequence extraction, it is possible to obtain $\mu$ supported on $\bigcap A_{n}$ and having finite $r$-energy: hence

$$
\forall r<1-s, \quad P\left(\operatorname{dim}_{H}\left(\bigcap A_{n}\right) \geqslant r\right)>0 .
$$

By definition $\mathcal{T}_{A}$ is the increasing union, for $R$ going to 0 , of $\bigcap_{n} A_{n}(R)$ : hence for all $r<1-s$ we have $P\left(\operatorname{dim}_{H}\left(\mathcal{T}_{A}\right) \geqslant r\right)>0$. Combining this and the zero-one result (Lemma 1) then proves that almost surely $\operatorname{dim}_{H}\left(\mathcal{T}_{A}\right) \geqslant 1-s$.

Second step: upper bound. This step is usually the easier one, but in the present case a complication arises due to the fact that the "non-intersection" event we consider at $B_{t}$ depends on the position of $B_{t}$ - which is not the case for instance in the case of cut-points [11]. This explains why we need one more argument, namely the continuity of $\xi: A \mapsto \xi(A)$.

Fix a nice set $A, \varepsilon>0, R>0$ and a sequence $\left(\lambda_{n}\right)_{n \geqslant 0}$ of positive numbers, tending slowly to 0 (in the following sense: for all positive $\eta, 2^{-\eta n}=o\left(\lambda_{n}\right)-$ for instance, take $\left.\lambda_{n}=1 / n\right)$. Now suppose some time $t$ is in $A_{n}$. With positive probability, the following happens:

$$
\left\{\begin{array}{l}
B_{\left[t-\lambda_{n} 2^{-n}, t+\lambda_{n} 2^{-n}\right]} \subset \mathcal{B}\left(B_{t}, \lambda_{n}^{1 / 2} 2^{-n / 2}\right), \\
\left|B_{t-2^{-n}}-B_{t}\right| \geqslant 2^{-n / 2}, \\
\left|B_{t+2^{-n}}-B_{t}\right| \geqslant 2^{-n / 2}, \\
\left(B_{\left[t-R, t-2^{-n}\right]} \cup B_{\left[t+2^{-n}, t+R\right]}\right) \cap \mathcal{B}\left(B_{t},(1-\varepsilon) 2^{-n / 2}\right)=\emptyset
\end{array}\right.
$$

(the first three conditions are a consequence of scaling, and the fourth one is the startseparation lemma, more precisely the weakened version of it as stated in Eq. (12)). Introduce $A^{\eta_{n}}=\left\{a z: a \in A, z \in \mathcal{B}\left(1, \eta_{n}\right)\right\}:$ we have

$$
\begin{aligned}
& P\left(\left(B_{\left[t-R, t-2^{-n}\right]}-B_{t}\right) \cap A^{\eta_{n}}\left(B_{\left[t+2^{-n}, t+R\right]}-B_{t}\right)=\emptyset \mid t \in A_{n}\right) \\
& \asymp \frac{2^{-n \xi\left(A^{\eta_{n}}\right) / 2}}{2^{-n \xi(A) / 2}}=2^{-n\left[\xi\left(A^{\eta_{n}}\right)-\xi(A)\right] / 2} .
\end{aligned}
$$

It is easy to see that under the previous conditions, if $t \in \mathcal{T}_{A^{\eta_{n}}}$, then every $t^{\prime} \in[t-$ $\left.\lambda_{n} 2^{-n}, t+\lambda_{n} 2^{-n}\right]$ is in $A_{n}$, as soon as $\eta_{n}>18 \lambda_{n} /(1-\varepsilon)$. From now on we shall assume that this holds, and that $\eta_{n} \rightarrow 0$. Putting these estimates together, we obtain the following (where $l$ is the Lebesgue measure on $\mathbb{R}$ ): for all interval $I$,

$$
P\left(l\left(A_{n} \cap I\right)>\lambda_{n} 2^{-n} \mid A_{n} \cap I \neq \emptyset\right) \geqslant c 2^{-n\left[\xi\left(A^{\eta_{n}}\right)-\xi(A)\right] / 2} .
$$

The Markov inequality then states that

$$
P\left(l\left(A_{n} \cap I\right)>\lambda_{n} 2^{-n}\right) \leqslant \frac{E\left(l\left(A_{n} \cap I\right)\right)}{\lambda_{n} 2^{-n}},
$$


and $E\left(l\left(A_{n} \cap I\right)\right) \asymp 2^{-n \xi(A) / 2} l(I)$. From this and (19) follows that

$$
P\left(A_{n} \cap I \neq \emptyset\right) \leqslant C \frac{2^{-n \xi(A) / 2} l(I)}{\lambda_{n} 2^{-n}} \frac{1}{2^{-n\left[\xi\left(A^{\eta_{n}}\right)-\xi(A)\right] / 2}} .
$$

By continuity of $\xi$, for large $n$ we have $\left|\xi\left(A^{\eta_{n}}\right)-\xi(A)\right|<\varepsilon$; by the hypothesis on $\lambda_{n}$, still for large $n$ we have $\lambda_{n} \geqslant 2^{-\varepsilon n / 2}$. Hence for large $n$ :

$$
P\left(A_{n} \cap I \neq \emptyset\right) \leqslant C 2^{\varepsilon n} 2^{-n \xi(A) / 2} \frac{l(I)}{2^{-n}} .
$$

Cover the interval $[0,1]$ with the $I_{k}^{n}=\left[k 2^{-n},(k+1) 2^{-n}\right]$, and let $X_{n}$ be the number of such intervals intersecting $A_{n}$. Then

$$
E\left(X_{n}\right)=\sum_{k} P\left(I_{k}^{n} \cap \mathcal{T}_{A} \neq \emptyset\right) \leqslant 2^{n} C 2^{\varepsilon n} 2^{-n \xi(A) / 2} \frac{l\left(I_{0}^{n}\right)}{2^{-n}} \leqslant C 2^{\varepsilon n} 2^{n[1-\xi(A) / 2]} .
$$

By another application of the Markov inequality,

$$
P\left(X_{n}>2^{n[1-\xi(A) / 2+2 \varepsilon]}\right) \leqslant C 2^{-\varepsilon n} .
$$

Hence by the Borel-Cantelli theorem, for sufficiently large $n, A_{n}$ is covered by at most $2^{n[1-\xi(A) / 2+2 \varepsilon]}$ intervals of length $2^{-n}-$ and this implies that $\operatorname{dim}_{H}\left(\bigcap A_{n}\right) \leqslant$ $1-\xi(A) / 2+2 \varepsilon$. Letting $\varepsilon$ tend to 0 then leads to (a.s.) $\operatorname{dim}_{H}\left(\bigcap A_{n}\right) \leqslant 1-\xi(A) / 2$. This is true for all $R>0$, hence remains true in the limit $R \rightarrow 0$ : together with the first step of the proof this gives (a.s.) $\operatorname{dim}\left(\mathcal{T}_{A}\right)=1-\xi(A) / 2$ hence $\operatorname{dim}\left(\mathcal{E}_{A}\right)=2-\xi(A)$.

Then, $\widetilde{\mathcal{E}}_{A}$ is contained in $\mathcal{E}_{A}$ and besides it contains every $\mathcal{E}_{A^{\eta}}$ for positive $\eta$ (with the previous notations): another use of the continuity of $\xi$ then gives $\operatorname{dim}_{H}\left(\widetilde{\mathcal{E}}_{A}\right)=$ $\operatorname{dim}_{H}\left(\mathcal{E}_{A}\right)=2-\xi(A)$.

As a consequence, we get a second result:

THEOREM 3. - If A is any nice subset of the complex plane, then the set of globally $A$-exceptional points, i.e. points $B_{t}$ satisfying

$$
\left(B_{[0, t)}-B_{t}\right) \cap A\left(B_{(t, 1]}-B_{t}\right)=\emptyset,
$$

has Hausdorff dimension $2-\xi(A)-$ and in particular it is a.s. non-empty for $\xi(A)<2$, and a.s. empty for $\xi(A)>2$.

Proof. - Again, extend $B$ to $\left(B_{t}\right)_{t \in \mathbb{R}}$ defined on the entire real line. The set $\mathcal{T}_{A}^{1}$ of $A$-exceptional times up to the scale $R=1$ (as was introduced previously) in $[0,1]$ is exactly the set of globally exceptional points. Therefore, the previous proof can be applied directly. The upper bound is immediate: since every globally exceptional point is locally exceptional we have $\operatorname{dim}_{H}\left(\mathcal{T}_{A}^{1}\right) \leqslant \operatorname{dim}_{H}\left(\mathcal{T}_{A}\right) \leqslant 1-\xi(A) / 2$ a.s.

The lower bound requires a little more work, indeed we do not have a zero-one law for the dimension of $\mathcal{T}_{A}^{1}$. It can be seen that in fact Eq. (17) can be refined, the proof 
being exactly the same, into the following (with the same notations as previously):

$$
\exists C>0 \forall r \in(0,1-s) \forall n>0 \quad E\left(\mathcal{E}_{r}\left(\mu_{n}\right)\right) \leqslant \frac{C}{1-(r+s)},
$$

where $C$ may only depend on $A$. Hence, with the same constant and for all $\lambda>1$ :

$$
P\left(\mathcal{E}_{r}\left(\mu_{n}\right) \leqslant \frac{\lambda C}{1-(r+s)}\right) \geqslant 1-\frac{1}{\lambda}
$$

one can then perform the subsequence extraction (cf. proof of Theorem 2) in a way which ensures that, for all $r$,

$$
P\left(\|\mu\|>0 \text { and } E_{r}(\mu) \leqslant \frac{\lambda C}{1-(r+s)}\right) \geqslant c,
$$

with $c>0$ and $\lambda>1$ independent of $r$. Moreover, $\mathcal{E}_{r}(\mu)$ being a non-decreasing function of $r$ (since the set $[0,1]$ is of diameter 1 ), we finally obtain, with positive probability, a mass measure $\mu$ supported on $\mathcal{T}_{A}$ satisfying

$$
\forall r<1-s, \quad \mathcal{E}_{r}(\mu) \leqslant \frac{\lambda C}{1-(r+s)}<\infty .
$$

Hence, with positive probability, $\operatorname{dim}_{H}\left(\mathcal{T}_{A}\right) \geqslant 1-s=1-\xi(A) / 2$, and combining this to the previous paragraph leads to

$$
P\left(\operatorname{dim}_{H}\left(\mathcal{T}_{A}\right)=1-\frac{\xi(A)}{2}\right)>0 .
$$

It is then possible to conclude using the same method as in [9, pp. 8-9].

\subsection{A remark about critical cases}

In cases where $\xi(A)=2$, the previous theorem is not sufficient to decide whether $A$-exceptional points exist. We shall see in the next paragraph that $\xi((-\infty, 0))=$ $\xi((0, \infty))=2$. In fact these two cases are very different:

PROPOSITION 4. -Almost surely, $\mathcal{E}_{A}$ is empty for $A=(0, \infty)$ and non-empty (with Hausdorff dimension 0 though) for $A=(-\infty, 0)$.

Proof. - The second point is easier: if $t$ is such that $\Re\left(B_{t}\right)$ is maximal in the path, then $B_{[0,1]}$ lies inside a half-plane whose border goes through $B_{t}$. Since a.s. $B_{t}$ is the only point having this real part, this proves that $\left(B_{s}-B_{t}\right) /\left(B_{s^{\prime}}-B_{t}\right)$ is never in $(-\infty, 0)$, which is precisely what we wanted.

The first point is more problematic. The method used to derive the value of $\xi$ for a wedge with end-point at the origin (cf. next paragraph) allows to prove the following: Let $\alpha$ and $\beta$ be in $(0,2 \pi)$, then the probability that, given independent paths $B^{1}$ and $B^{2}$ starting from the unit circle, there exist two wedges of angles $\alpha$ and $\beta$, and containing 
respectively $B^{1}$ and $B^{2}$ up to radius $R$, decreases as

$$
p_{R}(\alpha, \beta) \approx R^{-(\pi / \alpha+\pi / \beta)} .
$$

Hence, as soon as $\pi / \alpha+\pi / \beta$ is greater than 2 , there is a.s. no point $B_{t}$ on the path such that $B_{[0, t]}$ lies in a wedge of angle $\alpha$ and $B_{[t, 1]}$ lies in a wedge of angle $\beta$ (there is no "asymmetric two-sided cone point" of those angles on the path).

For all $\alpha \in(0, \pi)$, introduce $\alpha_{1}=2 \pi-\alpha$ and $\alpha_{2}$ as the biggest angle in $(0,2 \pi]$ satisfying $\pi / \alpha+\pi / \alpha_{2} \geqslant 2$. Note that $\alpha_{2}>\alpha_{1}$ : denote then

$$
\beta(\alpha)=\frac{\alpha_{1}+\alpha_{2}}{2} .
$$

Note that $\pi / \alpha+\pi / \beta(\alpha)>2$ and $\beta(\alpha)+\alpha>2 \pi$ for all $\alpha \in(0, \pi)$. From this follows that, almost surely, for all $\alpha \in(0, \pi) \cap \mathbb{Q}$, there is no asymmetric cone point with angles $\alpha$ and $\beta(\alpha)$.

Let now $A=(0, \infty)$ and suppose there is a point $B_{t}$ in $\mathcal{E}_{A}$. That is, there exist two half-lines starting from $B_{t}$ whose reunion separates $B_{[0, t]}$ from $B_{[t, 1]}$. Then we are in one of two cases:

- Either these half-lines form a straight line, i.e. there is a straight line cutting the path. This cannot happen, as recently proved by Bass and Burdzy [2] - and the proof is very difficult.

- Or there are disjoint wedges of angles $\alpha \in(0, \pi)$ and $2 \pi-\alpha$, each containing one part of the path. Then, there exists $\alpha_{0} \in \mathbb{Q}$ such that $\alpha_{0}>\alpha$ and $\beta\left(\alpha_{0}\right)>2 \pi-\alpha$, and $B_{t}$ is an asymmetric cone point with angles $\alpha_{0}$ and $\beta\left(\alpha_{0}\right)$. We just saw that such a point cannot exist.

Hence $\mathcal{E}_{A}=\emptyset$.

\section{Bounds and conjectures on the exponent function}

\subsection{Known exact values of $\xi$}

PROPOSITION 5. -

(i) $\xi(\{1\})=5 / 4$, hence for all $z \neq 0$ and $n>0$ :

$$
\xi\left(\left\{z \mathrm{e}^{2 \mathrm{i} k \pi / n}, k=1, \ldots, n\right\}\right)=\frac{5 n}{4} ;
$$

(ii) Letting $W_{\alpha}$ be a wedge of angle $0 \leqslant \alpha<2 \pi$ :

$$
\xi\left(W_{\alpha}\right)=\frac{4 \pi}{2 \pi-\alpha}
$$

in particular $\xi((0, \infty))=\xi((-\infty, 0))=2$;

Proof. - (i) The value of $\xi(\{1\})=5 / 4$ has recently been derived by Lawler, Schramm and Werner [15], and the proof is far beyond the scope of this paper. The result for all $n$ is then a straightforward consequence of Proposition 1, point (iv). 
(ii) Suppose $A=W_{\alpha}$ is centered around the positive axis, so that $A=\left\{r \mathrm{e}^{\mathrm{i} \theta}, r>0\right.$, $|\theta|<\alpha / 2\}$; introduce the symmetrical wedges $W_{\beta}^{\prime}=\left\{r \mathrm{e}^{\mathrm{i} \theta}, r>0,|\theta-\pi|<\beta / 2\right\}$. If $B^{1}$ stays in $W_{\pi-\alpha / 2}$ and $B^{2}$ remains in $W_{\pi-\alpha / 2}^{\prime}$, then $B^{1} \cap A B^{2}=\emptyset$ : The probability of staying in a wedge of angle $\beta$ until radius $R$ being strongly approximated by $R^{-\pi / \beta}$ (the exponent is obtained through the gambler's ruin estimate combined with the analyticity of the exponential function; the strong approximation is true but in fact not needed here, cf. [5]), we get a lower bound:

$$
p_{R}\left(W_{\alpha}\right) \geqslant c\left(R^{-\pi /(\pi-\alpha / 2)}\right)^{2}
$$

hence $\xi\left(W_{\alpha}\right) \leqslant 4 \pi /(2 \pi-\alpha)$.

Now remark that the condition $B^{1} \cap A B^{2}=\emptyset$ means that the complement of the paths contains an "hourglass", i.e. the union of two disjoint wedges of angle $\alpha / 2$. So introduce $\eta>0$ and a (finite) family $\left(S_{i}\right)_{1 \leqslant i \leqslant N}$ of hourglasses with angles $\alpha / 2-\eta$, such that any hourglass with angle $\alpha / 2$ contains one of the $S_{i}$. If $q_{R}(i)$ is the probability that the paths are separated from each other by $S_{i}$, then $p_{R}\left(W_{\alpha}\right) \leqslant \sum q_{R}(i)$. Noticing that if $\beta_{i}$ and $\beta_{i}^{\prime}$ are the angles of the wedges forming the complement of $S_{i}$, we obtain as previously $q_{R}(i) \asymp R^{-\pi / \beta_{i}-\pi / \beta_{i}^{\prime}}$, and optimizing this under the constraint $\beta_{i}+\beta_{i}^{\prime}=2 \pi-(\alpha-2 \eta)$ - where the greatest value is for $\beta=\beta^{\prime}$-we finally get the following estimate:

$$
p_{R}\left(W_{\alpha}\right) \leqslant C N R^{-2 \pi /(\pi+\eta-\alpha / 2)} .
$$

From this follows that $\xi\left(W_{\alpha}\right) \geqslant 4 \pi /(2 \pi+2 \eta-\alpha)$, and letting $\eta$ go to 0 then gives the conclusion - at least for $\alpha>0$. But in fact the same method still applies for $\alpha \geqslant 0$ : simply inflate the complement of the hourglass instead of introducing angle $\alpha / 2-\eta$, the fact that the wedges to consider may overlap does not change anything to the proof.

Remark. - If we denote $A^{\alpha}=\left\{z \mathrm{e}^{\mathrm{i} \theta}, z \in A,|\theta| \leqslant \alpha / 2\right\}$ (that is, $A$ "thickened" by an angle $\alpha$ ), then it can easily be proved that

$$
\xi\left(A^{\alpha}\right)=\frac{h_{A}(\alpha)}{2 \pi-\alpha}
$$

where $h_{A}$ is continuous (until the angle $\alpha_{0} \leqslant 2 \pi$ when $\xi\left(A^{\alpha}\right)$ tends to infinity), nondecreasing, and satisfies $h_{A}(0)=2 \pi \xi(A)$; in the wedge case, $h$ is constant.

\subsection{An upper bound for the exponent}

From continuity of $\xi$ and the exact value $\xi(\{1\})=5 / 4<2$, one can deduce that there are "pivoting points" of any sufficiently small angle on the Brownian path (that is, points around which one half of the path can rotate of a small angle without intersecting the other half - the associated $A$ being $\left.\mathcal{C}_{\alpha}=\left\{\mathrm{e}^{\mathrm{i} \theta}, \theta \in[0, \alpha]\right\}\right)$. The following proposition gives a (bad but) quantitative bound for such values of $\alpha$-without usage of the exact value for $\alpha=0$ : 
Proposition 6. - For all positive $\alpha$, we have the following upper bound:

$$
\xi\left(\mathcal{C}_{\alpha}\right) \leqslant \frac{4 \pi}{2 \pi-\alpha}\left[1-\frac{(\log 2)^{2}}{4 \pi^{2}}\right] .
$$

Proof. - The proof is adapted from [22], where an upper bound for the classical disconnection exponent for one path, i.e. $\xi(1,0)$, was obtained. The method is the following: First, estimate the extremal length of a strip bounded by Lipschitz functions; then describe a sufficiently large subset of $E_{R}$, using such strips, and use the previous estimate to derive a bound for $P\left(E_{R}\right)$.

LEMMA. - Let $f$ be a continuous, M-Lipschitz function on $\mathbb{R}$, satisfying $f(x)+$ $f(-x)=2 f(0)$ for all $x$, and let $\beta>0$. Introduce the strip of width $\beta$ and length $2 r$ around $f$ as

$$
\mathcal{B}_{f}^{\beta}(r)=\left\{x+\mathrm{i} y:|x|<r,|y-f(x)|<\frac{\beta}{2}\right\} ;
$$

let $W$ be a planar Brownian path starting at if $(0)$, and denote $A_{f}^{\beta}(r)$ the event that the point $x+i y$ where $W$ first reaches $\partial \mathcal{B}_{f}^{\beta}(r)$ satisfies $|x|=r$ (i.e., $W$ exits $\mathcal{B}$ by one of the vertical parts of its boundary). Then

$$
P\left(A_{f}^{\beta}(r)\right) \geqslant \frac{1}{\pi} \exp \left[-\frac{\pi r}{\beta}\left(1+M^{2}\right)\right] .
$$

Proof. - This is an easy consequence of the following estimate, which can be found in [1] and is a consequence of Proposition 9: If $L$ is the extremal distance between both vertical parts of $\partial \mathcal{B}$ in $\mathcal{B}$, then

$$
L \leqslant \frac{2 r}{\beta}\left(1+M^{2}\right)
$$

using this together with the classical estimate for Brownian motion in a strip provides the right estimate.

For the rest of this proof, we shall consider paths in the logarithmic space, denoted by the letter $W$; the actual path $B$ is obtained from $W$ by applying the exponential map - conformal invariance of Brownian motion then proves that $B$ is a Brownian path. Let $f$ be a function such as in the lemma: it is clear that if $W^{1}$ remains in $\mathcal{B}_{f}^{\pi}(r)$ and $W^{1}$ stays in $\mathcal{B}_{f+\pi}^{\pi}(r)$, then $B^{1}$ and $B^{2}$ do not intersect up to the first time they reach radius $\mathrm{e}^{r}$ or $\mathrm{e}^{-r}$. Together with the fact that $P\left(A_{f}^{\pi}(r)\right)=P\left(A_{f+\pi}^{\pi}(r)\right)$, this leads to $P\left(E_{R}(\{1\})\right) \geqslant\left(P\left(A_{f}^{\pi}(\log R)\right) / 2\right)^{2}$, hence using the lemma:

$$
P\left(E_{R}(\{1\})\right) \geqslant c R^{-2\left(1+M^{2}\right)} .
$$

Doing the same with strips of width $\beta=\pi-\alpha / 2$ (for which it can be seen that $B^{1}$ and $B^{2}$ can rotate around 0 by an angle at least $\alpha / 2$ in each direction) leads to

$$
P\left(E_{R}\left(\mathcal{C}_{\alpha}\right)\right) \geqslant c \exp \left[-\frac{4 \pi}{2 \pi-\alpha}\left(1+M^{2}\right) \log R\right]
$$


hence, letting $f=0$, a first bound on the exponent:

$$
\xi\left(\mathcal{C}_{\alpha}\right) \leqslant \frac{4 \pi}{2 \pi-\alpha}
$$

(this is also a direct consequence of $\mathcal{C}_{\alpha} \subset W_{\alpha}$ and the exact value of $\xi\left(W_{\alpha}\right)$, which happens to be precisely the upper bound we just obtained). Note that the bound is never less than 2, hence we proved nothing useful yet.

We now want to consider families of strips. Keep $\beta=\pi-\alpha / 2$ and fix $\gamma>0$; let $U_{N}=\{ \pm 1\}^{N}$ and for $u \in U_{N}$ let $f_{u}$ be constructed as follows:

- $f_{u}(0)=0$, and for $1 \leqslant n \leqslant N, f_{u}(n \gamma)=\frac{\beta}{2} \sum_{k=1}^{n} u_{k}$;

- $f$ is affine on each $[n \gamma,(n+1) \gamma]$, satisfies $f_{u}(x)=f_{u}(N \gamma)$ for all $x>N \gamma$ and $f_{u}(-x)=-f_{u}(x)$ for all $x$.

Then for $u \neq u^{\prime}$ the intersection of $\mathcal{B}_{f_{u}}^{\beta}$ and $\mathcal{B}_{f_{u^{\prime}}}^{\beta}$ is not connected, hence $A_{f_{u}}^{\beta}$ and $A_{f_{u^{\prime}}}^{\beta}$ are disjoint. This leads to

$$
P\left(E_{R}\left(\mathcal{C}_{\alpha}\right)\right) \geqslant c \sum_{u \in U_{N}} \exp \left[-\frac{2 \pi}{\beta}\left(1+(\beta / 2 \gamma)^{2}\right) \log R\right]
$$

for all $N$, where $R=\mathrm{e}^{N \gamma}$. Then using $P\left(E_{R}\left(\mathcal{C}_{\alpha}\right)\right) \asymp R^{-\xi\left(\mathcal{C}_{\alpha}\right)}$, noticing that all the terms of the sum are equal (there are $2^{N}$ of them) and applying a logarithm:

$$
\xi\left(\mathcal{C}_{\alpha}\right) N \gamma \leqslant \frac{2 \pi}{\beta}\left(1+(\beta / 2 \gamma)^{2}\right) N \gamma-N \log 2-\log c .
$$

Divide by $N \gamma$ and let $N$ go to infinity to obtain

$$
\xi\left(\mathcal{C}_{\alpha}\right) \leqslant \frac{\pi \beta}{2}\left(\frac{1}{\gamma}\right)^{2}-\log 2\left(\frac{1}{\gamma}\right)+\frac{2 \pi}{\beta} .
$$

This is true for all $\gamma>0$; the optimal value is $\gamma=\pi \beta / \log 2$, leading to

$$
\xi\left(\mathcal{C}_{\alpha}\right) \leqslant \frac{4 \pi}{2 \pi-\alpha}\left[1-\frac{(\log 2)^{2}}{4 \pi^{2}}\right],
$$

which is precisely what we wanted.

Remark. - The same proof gives a bound on $\xi(A)$ if $A$ is included in a small ball centered at 1 , as a function of the radius. But since it does not make use of the value of $\xi(\{1\})$, no modulus of continuity for $\xi$ can be obtained this way. Cf. however Eq. (23) for another bound, which does provide such a modulus but is not quantitative.

As a consequence of this bound, we obtain the following

THEOREM 4. - For all $\alpha<\log ^{2} 2 / 2 \pi$, the following holds: With probability 1 , the set of local pivoting points of angle $\alpha$ on a planar Brownian path is non-empty and has a positive Hausdorff dimension. 


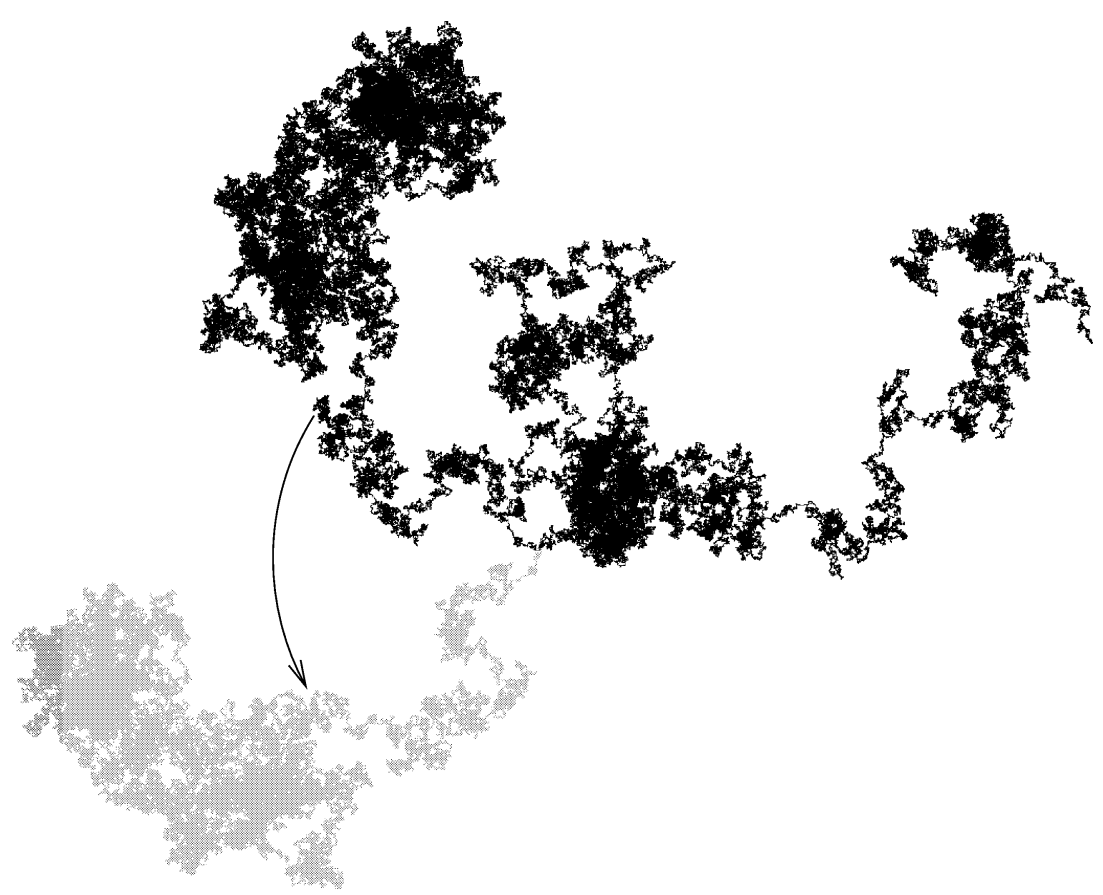

Fig. 1. A pivoting point of angle $\pi / 2$ (in gray is the image of one half of the path by a rotation of angle $+\pi / 2$ ).

Remark. - The bound given in the theorem $\left(\log ^{2} 2 / 2 \pi \simeq 0.076\right)$ is certainly not the best one; simulations suggest that there are pivoting points of any angle less than $3 \pi / 4 \simeq 2.356$ - cf. next subsection for details and Fig. 1 for a picture of a pivot of angle $\pi / 2$. In particular, the maximal angle is conjectured to be greater than $2 \pi / 3$, and this seems to indicate that a discrete analogue of (local) pivoting points will appear on the exploration process of a critical percolation cluster on the triangular lattice [20,21].

\subsection{Conjectured and experimental values}

Some exact values of $\xi(A)$ are known, cf. Subsection 4.2. However, heuristic arguments seem to indicate that the formula giving the exponent for wedges is close to apply in other cases such as notably the "weak pivot" exponent, namely:

$$
\xi\left(\left\{1, \mathrm{e}^{\mathrm{i} \theta}\right\}\right) \simeq \frac{5 \pi / 2}{2 \pi-\theta}
$$

for all $\theta \in[0, \pi]-$ corresponding to a continuous version of Proposition 1, point (iv). This is confirmed by simulations, at least for $\theta=\pi / 2$ and $\theta=\operatorname{arctg}(3 / 4)$ (cf. Table 1 ), based on the following

CONJECTURE. - Let $A$ be a bounded, non-empty subset of $\mathbb{Z}^{2} \backslash\{0\}$; let $B^{1}$ and $B^{2}$ be independent Brownian paths starting respectively from 0 and 1 , and $S^{1}$ and $S^{2}$ be independent standard random walks starting respectively from 0 and $(a, 0)$ with a 
Table 1

Some simulated values of $\xi$ (100000-step walks - exponents marked with a star are obtained after a non-rigorous correction)

\begin{tabular}{ccccc}
\hline \multicolumn{1}{c}{$A$} & $\begin{array}{c}\text { Conjectured } \\
\text { exponent }\end{array}$ & $\begin{array}{c}\text { Number } \\
\text { of samples }\end{array}$ & $\begin{array}{c}\text { Computed } \\
\text { exponent }\end{array}$ & $\begin{array}{c}\text { Relative } \\
\text { error }(\%)\end{array}$ \\
\hline$\{ \pm 1\}$ & $\sim 2.5$ & $2.6 \times 10^{9}$ & 2.501293 & +0.05 \\
$\{1, \mathrm{i}\}$ & $\sim 5 / 3$ & $3.0 \times 10^{8}$ & 1.662239 & -0.27 \\
& & & $1.668242 *$ & +0.09 \\
$\{5,4+3 \mathrm{i}\}$ & $\sim 1.392679$ & $1.2 \times 10^{6}$ & 1.382311 & -0.74 \\
& & & $1.394610 *$ & +0.14 \\
$\{5,4+3 \mathrm{i}, 5 \mathrm{i}\}$ & $\sim 5 / 3$ & $1.6 \times 10^{7}$ & 1.662964 & -0.22 \\
& & & $1.665650 *$ & -0.06 \\
\hline
\end{tabular}

sufficiently large (so as not to make the probability in the formula equal to 0). Then,

$$
P\left(B_{[0, T]}^{1} \cap A B_{[0, T]}^{2}=\emptyset\right) \asymp P\left(S_{[0, T]}^{1} \cap A S_{[0, T]}^{2}=\emptyset\right) \asymp T^{-\xi(A) / 2} .
$$

Proof. - There is no known direct proof of the existence of a non-intersection exponent for random walks, the only way to obtain the desired behaviour is coupling with Brownian motion - cf. [12]. The present generalization can certainly be obtained in a similar way, note however that walks appear that are not standard simple random walks but take steps in $\{a, \mathrm{i} a,-a,-\mathrm{i} a\}$ for some $a \in \mathbb{C} \cap \mathbb{Z}^{2}$; exponents for such walks are the same as for SRW's (cf. [3]), but strong approximation in not yet proved.

The most severe restriction is the assumption that $A \subset \mathbb{Z}^{2} \backslash\{0\}$, in particular simulations cannot (yet) be performed if $A$ is connected, except for very special cases such as wedges (where the exact exponent is known). However homogeneity can sometimes be used when $A \in \mathbb{Q}^{2}$ (as for $A=\{5,4+3 \mathrm{i}\}$ which has the same exponent as $\left\{1, \mathrm{e}^{\mathrm{i} \theta}\right\}$ for $\left.\theta=\operatorname{arctg}(3 / 4)\right)$.

\section{Acknowledgement}

I wish to thank Wendelin Werner for suggesting that I look for pivoting points on the planar Brownian curve and for never refusing help and advice.

\section{Appendix A}

\section{A.1. Sub-additivity}

The following proposition is well known and included here only for completeness (note however that the bounds are not asymptotic and that the constants are exactly known, which is needed to derive continuity of $\xi$ ). A proof can be found, e.g., in [4, Lemma 6.1.11]. 
Proposition 7 (Sub-additivity). - Let $f:[1, \infty) \rightarrow(0, \infty)$ be some function such that:

- $f$ is bounded and bounded away from 0 on any $[0, l], l>0$;

- There exist $\varepsilon, A, c$ and $C$ in $(0, \infty)$ such that for all $t \geqslant 1, c t^{-A} \leqslant f(t) \leqslant C t^{-\varepsilon}$;

- There exist $0 \leqslant c_{-} \leqslant c_{+} \leqslant \infty$, at least one of which finite and positive, such that

$$
\forall t, t^{\prime} \in[1, \infty) \quad c_{-} f(t) f\left(t^{\prime}\right) \leqslant f\left(t t^{\prime}\right) \leqslant c_{+} f(t) f\left(t^{\prime}\right) .
$$

Then, there is $a \xi>0$ such that $f(t) \approx t^{-\xi}$. Moreover, for all $t \geqslant 1$,

$$
c_{+}^{-1} t^{-\xi} \leqslant f(t) \leqslant c_{-}^{-1} t^{-\xi} .
$$

In particular, if both $c_{-}$and $c_{+}$are in $(0, \infty)$ we get strong approximation $f(t) \asymp t^{-\xi}$.

\section{A.2. Extremal distance}

Many of the known estimates for exponents (apart from cases where the exact value in known - such as the exponent of a cone here, and the intersection exponents in the halfplane in [14]) come from the corresponding estimates for Brownian paths in rectangles, using conformal invariance. The introduction of extremal distance generalizes the notion of aspect ratio of a rectangle and hence provides a natural parameter in this process.

THEOREM AND DEFINITION. - Let $\Omega$ be an open, bounded, simply connected subset of $\mathbb{C}$, the frontier of which (oriented in the usual direct sense) is a Jordan curve $\gamma:[0,1] \rightarrow \partial \Omega$; fix four real numbers $0<a<b<c<d<1$. Then there exist $a$ unique positive real number $L$ and a unique conformal map $\Phi: \Omega \rightarrow(0, L) \times(0,1)$, with natural extension to $\bar{\Omega}$, such that $\Phi(\gamma(a))=\mathrm{i}, \Phi(\gamma(b))=0, \Phi(\gamma(c))=L$ and $\Phi(\gamma(d))=L+\mathrm{i}$.

$L$ is called extremal distance between $\partial_{1}=\gamma([a, b])$ and $\partial_{2}=\gamma([c, d])$ in $\Omega$; it is denoted $\mathrm{d}_{\Omega}\left(\partial_{1}, \partial_{2}\right)$.

Proof. - For the proof of this result, and much more about conformal maps and related topics (including the proofs of Propositions 8 and 9), cf. [1].

The extremal distance between both sides of length $a$ in an $a \times b$ rectangle is $b / a$. By the analyticity of the logarithm in $\mathbb{C} \backslash(-\infty, 0]$, if $\Omega=\left\{\rho \mathrm{e}^{\mathrm{i} \theta}: r<\rho<R, 0<\theta<\alpha\right\}$ with $0<r<R<\infty$ and $0<\alpha<2 \pi$, then the extremal distance in $\Omega$ between both circle arcs is $\alpha^{-1} \log (R / r)$. Finally, if $L$ is the extremal distance in $\Omega$ between two connected parts $\partial_{1}$ and $\partial_{2}$ of $\partial \Omega$, then the extremal distance between the two components of $\partial \Omega \backslash\left(\partial_{1} \cup \partial_{2}\right)$ is $L^{-1}$.

Proposition 8. - Let $\rho: \Omega \rightarrow[0, \infty)$ be a continuous function, and denote $A_{\rho}(\Omega)=\iint_{\Omega} \rho^{2}$ and for any continuous arc $\gamma$ in $\Omega, L_{\rho}(\gamma)=\int_{\gamma} \rho(z)|\mathrm{d} z|$ (this defines the Riemannian metric associated with $\rho$ ). Then we have, thus giving a justification to the term extremal length, the following characterization of $\mathrm{d}_{\Omega}$ :

$$
\mathrm{d}_{\Omega}\left(\partial_{1}, \partial_{2}\right)=\operatorname{Sup}_{\rho} \operatorname{Inf}_{\gamma: \partial_{1} \rightsquigarrow \partial_{2}} \frac{L_{\rho}(\gamma)^{2}}{A_{\rho}(\gamma)}
$$


(where $\gamma: \partial_{1} \rightsquigarrow \partial_{2}$ means that $\gamma$ is a continuous path in $\Omega$ with first and second endpoints respectively in $\partial_{1}$ and $\partial_{2}$ ).

In many cases, it is sufficient to apply this with a finite family of $\rho$ 's to obtain a fairly good lower bound for $\mathrm{d}_{\Omega}-$ usually even $\rho=1$, i.e. taking the Euclidean metric, is sufficient. Another estimate for $d_{\Omega}$ is the following:

Proposition 9. - Let $L$ be a positive real number and $f_{1}, f_{2}:[0, L] \rightarrow \mathbb{R}$ be two continuous functions such that for all $t$ in $[0, L]$ we have $f_{1}(t)<f_{2}(t)$. Introduce $\Omega=\left\{x+\mathrm{i} y: 0<x<L, f_{1}(x)<y<f_{2}(x)\right\}$, and let $\partial_{1}$ and $\partial_{2}$ stand for the vertical components of $\partial \Omega$. Then

$$
\mathrm{d}_{\Omega}\left(\partial_{1}, \partial_{2}\right) \geqslant \int_{0}^{L} \frac{\mathrm{d} t}{f_{2}(t)-f_{1}(t)} .
$$

Moreover, if $f_{1}$ has a continuous derivative and $f_{2}=f_{1}+a$, then

$$
\mathrm{d}_{\Omega}\left(\partial_{1}, \partial_{2}\right) \leqslant \frac{L}{a}\left[1+\left\|f_{1}^{\prime}\right\|_{\infty}^{2}\right]
$$

\section{A.3. Some topological tools}

In this section, all sets considered will be assumed non-empty.

DEFINITION. - If $A$ is a subset of the set $\mathbb{C}$ of complex numbers (or of any Banach space), note

$$
V_{r}(A)=\{x \in \mathbb{C}: \mathrm{d}(x, A)<r\}=A+\mathcal{B}(0, r) ;
$$

if $A$ and $B$ are two bounded subsets of $\mathbb{C}$, introduce the Hausdorff distance between $A$ and $B$ as

$$
\mathrm{d}_{H}(A, B)=\operatorname{Inf}\left\{r: A \subset V_{r}(B), B \subset V_{r}(A)\right\} .
$$

It is easy to see that $\mathrm{d}_{H}$ is nonnegative and satisfies the triangle inequality (namely $\mathrm{d}_{H}(A, B) \leqslant \mathrm{d}_{H}(A, C)+\mathrm{d}_{H}(C, B)$ for any $\left.A, B, C\right)$; moreover $\mathrm{d}_{H}(A, B)=0$ if and only if $\bar{A}=\bar{B}$. Hence, $\mathrm{d}_{H}$ defines a metric topology on the set of compact subsets of $\mathbb{C}$, known as the Hausdorff topology.

We need the following standard property about the Hausdorff topology on the subsets of some fixed set, describing the compact case:

Proposition 10. - Let $K$ be a compact subset of $\mathbb{C}$. Then the set $\mathcal{P}_{c}(K)$ of all (non-empty) closed subsets of $K$, equipped with the topology induced by the Hausdorff distance, is compact.

Remark. - It is still true (and the proof is basically the same) that for any complete space $E$ the set $\mathcal{P}_{c}(E)$ is complete. Moreover, if $E$ is locally compact, so is $\mathcal{P}_{c}(E)$. However, it is generally not bounded, hence not compact. 


\section{REFERENCES}

[1] L.V. Ahlfors, Conformal Invariants, in: Topics in Geometric Function Theory, McGrawHill, New York, 1973.

[2] R.F. Bass, K. Burdzy, Cutting Brownian Paths, in: Mem. Amer. Math. Soc., Vol. 657, American Mathematical Society, 1999.

[3] M.C. Cranston, T.S. Mountford, An extension of a result of Burdzy and Lawler, Probab. Theory Related Fields 89 (1991) 487-502.

[4] A. Dembo, O. Zeitouni, Large Deviations Techniques and Applications, 2nd Edition, Springer, 1998.

[5] S.N. Evans, On the Hausdorff dimension of Brownian cone points, Math. Proc. Cambridge Philos. Soc. 98 (1985) 343-353.

[6] J.-P. Kahane, Some Random Series of Functions, 2nd Edition, in: Cambridge Stud. Adv. Math., Vol. 5, Cambridge University Press, 1993.

[7] R. Kaufman, Une propriété métrique du mouvement brownien, C. R. Acad. Sci., Sér. A 268 (1969) 727-728.

[8] G.F. Lawler, The dimension of the frontier of planar Brownian motion, Electron Comm. Probab. 1 (1996) 29-47.

[9] G.F. Lawler, Hausdorff dimension of cut points for Brownian motion, Electron. J. Probab. 1 (1996) 1-20.

[10] G.F. Lawler, Strict concavity of the intersection exponent for Brownian motion in two and three dimensions, Math. Phys. Electron. J. 4 (1998).

[11] G.F. Lawler, Geometric and fractal properties of Brownian motion and random walk paths in two and three dimensions, in: Random Walks (Budapest 1998), in: Bolyai Soc. Math. Stud., Vol. 9, 1999, pp. 219-258.

[12] G.F. Lawler, E.E. Puckette, The intersection exponent for simple random walks, Comb. Prob. Comp. 9 (2000) 441-464.

[13] G.F. Lawler, O. Schramm, W. Werner, The dimension of the Brownian frontier is 4/3, Math. Rev. Lett. 8 (2001) 13-24.

[14] G.F. Lawler, O. Schramm, W. Werner, Values of Brownian intersection exponents I: Halfplane exponents, Acta Math. 187 (2001) 237-273.

[15] G.F. Lawler, O. Schramm, W. Werner, Values of Brownian intersection exponents II: Plane exponents, Acta Math. 187 (2001) 275-308.

[16] G.F. Lawler, O. Schramm, W. Werner, Analyticity of intersection exponents for planar Brownian motion, Acta Math. 188 (2002), to appear.

[17] G.F. Lawler, O. Schramm, W. Werner, Values of Brownian intersection exponents III: Twosided exponents, Ann. Inst. H. Poincaré Probab. Statist. 38 (2002) 109-123.

[18] G.F. Lawler, W. Werner, Intersection exponents for planar Brownian motion, Ann. Probab. (1999) 1601-1642.

[19] J.-F. Le Gall, Some properties of planar Brownian motion, in: École d'été de Probabilités de Saint-Flour XX-1990, in: Lecture Notes in Math., Vol. 1527, Springer, 1992.

[20] O. Schramm, Scaling limits of loop-erased random walks and uniform spanning trees, Israel J. Math. 118 (2000) 221-288.

[21] S. Smirnov, Critical percolation in the plane: Conformal invariance, Cardy's formula, scaling limits, C. R. Acad. Sci. Paris Sér. I Math. 333 (2001) 239-244.

[22] W. Werner, On Brownian disconnection exponents, Bernoulli 1 (1995) 371-380.

[23] W. Werner, Bounds for disconnection exponents, Electron. Comm. Probab. 1 (1996) 19-28.

[24] W. Werner, Critical exponents, conformal invariance and planar Brownian motion, in: Proceedings of the 3rd European Mathematical Congress, Birkhäuser, 2000. 